\title{
The dark phases of the N2HDM
}

\author{
Isabell Engeln, ${ }^{a}$ Pedro Ferreira, ${ }^{b, c}$ M. Margarete Mühlleitner, ${ }^{a}$ Rui Santos ${ }^{b, c}$ and \\ Jonas Wittbrodt ${ }^{d}$ \\ ${ }^{a}$ Institute for Theoretical Physics, Karlsruhe Institute of Technology, \\ 76128 Karlsruhe, Germany \\ ${ }^{b}$ Centro de Física Teórica e Computacional, Faculdade de Ciências, Universidade de Lisboa, \\ Campo Grande, Edifício C8 1749-016 Lisboa, Portugal \\ ${ }^{c} I S E L$ - Instituto Superior de Engenharia de Lisboa, Instituto Politécnico de Lisboa, \\ 1959-007 Lisboa, Portugal \\ ${ }^{d}$ Department of Astronomy and Theoretical Physics, Lund University, \\ Sölvegatan 14A, 223 62 Lund, Sweden \\ E-mail: anna.engeln@student.kit.edu, pmmferreira@fc.ul.pt, \\ margarete.muehlleitner@kit.edu, rasantos@fc.ul.pt, \\ jonas.wittbrodt@thep.lu.se
}

ABStract: We discuss the dark phases of the Next-to-2-Higgs Doublet model. The model is an extension of the Standard Model with an extra doublet and an extra singlet that has four distinct $\mathrm{CP}$-conserving phases, three of which provide dark matter candidates. We discuss in detail the vacuum structure of the different phases and the issue of stability at tree-level of each phase. Taking into account the most relevant experimental and theoretical constraints, we found that there are combinations of measurements at the Large Hadron Collider that could single out a specific phase. The measurement of $h_{125} \rightarrow \gamma \gamma$ together with the discovery of a new scalar with specific rates to $\tau^{+} \tau^{-}$or $\gamma \gamma$ could exclude some phases and point to a specific phase.

Keywords: Beyond Standard Model, Higgs Physics

ARXIV EPRINT: 2004.05382 


\section{Contents}

1 Introduction 1

2 The N2HDM 3

2.1 The broken phase (BP) 6

$\begin{array}{lll}2.2 & \text { The dark doublet phase (DDP) } & 7\end{array}$

2.3 The dark singlet phase (DSP) 9

$\begin{array}{ll}2.4 \text { The fully dark phase (FDP) } & 10\end{array}$

$\begin{array}{lll}3 & \text { Neutral vacua stability } & 10\end{array}$

4 Parameter scans and constraints $\quad 15$

5 The different phases at the LHC and future colliders 18

$\begin{array}{lll}5.1 & h_{125} \text { coupling measurements } & 18\end{array}$

$\begin{array}{ll}5.2 & \text { Search for new scalars }\end{array}$

6 Conclusions $\quad 22$

A Dark doublet phase $\quad 23$

A.1 Triple-Higgs couplings 24

B Dark singlet phase $\quad 24$

B.1 Triple-Higgs couplings 25

\section{Introduction}

After the discovery of the Higgs boson [1,2] a large number of extensions of the Standard Model (SM) were explored at the Large Hadron Collider (LHC) by searching both for new particles and for deviations in the Higgs couplings to the remaining SM particles. However, not only are there no direct hints of new physics so far but all Higgs rates are in very good agreement with the SM predictions. Still, there is clear evidence of new physics, and in particular the existence of Dark Matter (DM) which will be the subject of the particular extension of the SM to be discussed in this work.

The existence of DM manifests itself in gravitational effects to baryon acoustic oscillations in the cosmic microwave background radiation [3], which has shown that the relic abundance of DM in the Universe is about 26\% [4-6]. Although there is no indication about the nature of DM, it is clear that a particle with a mass around the scale of electroweak symmetry breaking and an interaction cross section with the SM particles of the order of the weak force processes can account for the observed relic abundance as 
well as for structure formation. These candidates are called Weakly Interacting Massive Particles (WIMPs).

When considering extensions of the SM with a DM candidate one needs to take into account all the presently available constraints. In order to have an SM-like Higgs boson of $125 \mathrm{GeV}$ and a scalar DM candidate, the simplest extension of the SM is just the addition of a singlet field either real or complex [7-9]. The additional singlet is neutral with respect to the SM gauge groups and DM is stabilised by a symmetry. The next simplest extension that ensures $\rho=1$ at tree level is the popular Inert Doublet Model (IDM) [10-13], a 2Higgs Doublet Model where only one of the doublets acquires a vacuum expectation value (VEV). The dark doublet (and the dark Higgs) is protected by a $\mathbb{Z}_{2}$ symmetry. The new dark sector contains two charged and two neutral fields, the lightest of which is the dark matter candidate.

The Next-to-2-Higgs-Doublet Model (N2HDM) [14-17], is an extension of the scalar sector of the SM by one doublet and one real singlet. In the particular version of doublet plus singlet extension that we will be studying, two $\mathbb{Z}_{2}$ symmetries are enforced. Depending on the pattern of symmetry breaking one ends up with a model with no dark matter candidates, or a model with one or two dark matter particles. When unbroken, one of the $\mathbb{Z}_{2}$ symmetries stabilises the additional doublet, while the other stabilises the additional singlet. Therefore, the model has four distinct phases: one with no DM, one with a singletlike DM particle, one with a doublet-like DM candidate and finally one with two DM candidates. We call the phase with singlet-like DM phase [15] the Dark Singlet Phase (DSP), the doublet-like phase is called Dark Doublet Phase (DDP) and the SM-like phase with the two unbroken $\mathbb{Z}_{2}$ symmetries is designated Full Dark Matter Phase (FDP).

Because the two symmetries are exact, both spontaneous and explicit CP-violation cannot occur in any of the phases. In the DSP, the visible sector resembles the $\mathbb{Z}_{2}$ symmetric $2 \mathrm{HDM}$ and the absence of the soft breaking $m_{12}^{2}$ term precludes the possibility of CPviolation in the scalar sector, since no complex phase can be produced in the doublets when spontaneous symmetry breaking occurs; in the DDP, even with a complex singlet VEV CPviolation would not be possible because the singlet has zero isospin and zero hypercharge and therefore only couples to the scalar doublets in the Higgs potential - that singlet phase could in fact be removed via a field redefinition from the whole lagrangian and thus has no physical implications. Since in this work we will not deal with CP violation, explicit or spontaneous, let it be clear that by "phase" we will always mean a given vacuum of the model, with different broken symmetries, rather than any complex phase of the parameters or of VEVs of the fields.

In this work, we compare the three N2HDM dark phases and wherever relevant we also include the Broken Phase (BP), where the vacuum breaks both $\mathbb{Z}_{2}$ symmetries and there is no dark matter candidate. The DSP and DDP have additional scalar particles that mix with the CP-even scalar from the SM doublet giving rise to new final states. We will discuss how to phenomenologically distinguish these two phases. The comparison between the three phases (and between each of them and the SM) can only be performed in the $125 \mathrm{GeV}$ Higgs $\left(h_{125}\right)$ decays and couplings to the remaining SM particles. This is accomplished by studying the decay $h_{125} \rightarrow \gamma \gamma$ where an extra loop of charged Higgs scalars - either from the dark or from the visible phases — contributes. 
There are many reasons to study models with dark matter candidates beyond minimal models. The first and very interesting point that motivated this study is that we have for the exact same model, different phases with very different phenomenology. The model may have no dark matter candidates, a singlet-like dark matter candidate, a doublet-like one and finally a phase that is not at all present in minimal models with two dark matter candidates. In all models there are additional scalars that can be either in the visible sector or in the dark sector. There are at least 2 neutral Higgs bosons (except for the FDP) in the visible sector and it allows for the study of the effects of singlet-like degrees of freedom. Moreover, due to its enlarged Higgs sector Higgs pair production with two different Higgs bosons in the final state is possible together with Higgs-to-Higgs cascade decays. As discussed in our previous work [18] the broken phase of the N2HDM has a quite different phenomenology from other models with more than two neutral scalars, like singlet extensions (CxSM), CP-violating doublet extensions (like the complex 2HDM) and also the NMSSM. Finally the additional scalars in the visible sector can play an important role by providing additional annihilation channels for DM. In that way DM mass regions that are excluded in minimal models are not excluded here. We will show - taking into account all available experimental data - that the allowed dark matter mass region in the different phases is not at all the same. The dark matter mass range in turn impacts visible sector observables, such as (semi-)invisible decays of the visible scalars. Many of the resulting signatures can only be studied in non-minimal DM models. The study of non-minimal models of DM with distinct phenomenology in the visible and the dark sector is thus important in order to leave no stone unturned in LHC searches for new physics.

The structure of the paper is as follows. We start by defining and describing the model and its phases in section 2 . In section 3 we study the coexistence of minima of different phases, and analyse the vacuum structure of the model. In the following section 4 we present the experimental and theoretical constraints imposed on the model. In section 5 we discuss how the different phases can be probed at the Large Hadron Collider and add a brief discussion on future colliders. Finally we conclude in section 6 . The relations between the physical quantities at each phase and the input parameters of the model are shown in the appendices.

\section{The N2HDM}

The N2HDM [14-17] is an extension of the SM, where a complex $\mathrm{SU}(2)_{L}$ doublet $\Phi_{2}$ with hypercharge $Y=+1$ and a real $\mathrm{SU}(2)_{L}$ singlet $\Phi_{S}$ with $Y=0$ are added to the SM field content. In this work we will consider the most general renormalisable scalar potential invariant under two $\mathbb{Z}_{2}$ symmetries: the first is

$$
\mathbb{Z}_{2}^{(1)}: \quad \Phi_{1} \rightarrow \Phi_{1}, \quad \Phi_{2} \rightarrow-\Phi_{2}, \quad \Phi_{S} \rightarrow \Phi_{S},
$$

while the second is

$$
\mathbb{Z}_{2}^{(2)}: \quad \Phi_{1} \rightarrow \Phi_{1}, \quad \Phi_{2} \rightarrow \Phi_{2}, \quad \Phi_{S} \rightarrow-\Phi_{S} .
$$


Both symmetries are exact and - if not spontaneously broken - will give rise to DM candidates after electroweak symmetry breaking (EWSB). The potential reads

$$
\begin{aligned}
V_{\text {Scalar }}= & m_{11}^{2} \Phi_{1}^{\dagger} \Phi_{1}+m_{22}^{2} \Phi_{2}^{\dagger} \Phi_{2}+\frac{\lambda_{1}}{2}\left(\Phi_{1}^{\dagger} \Phi_{1}\right)^{2}+\frac{\lambda_{2}}{2}\left(\Phi_{2}^{\dagger} \Phi_{2}\right)^{2} \\
& +\lambda_{3} \Phi_{1}^{\dagger} \Phi_{1} \Phi_{2}^{\dagger} \Phi_{2}+\lambda_{4} \Phi_{1}^{\dagger} \Phi_{2} \Phi_{2}^{\dagger} \Phi_{1}+\frac{\lambda_{5}}{2}\left[\left(\Phi_{1}^{\dagger} \Phi_{2}\right)^{2}+\text { h.c. }\right] \\
& +\frac{1}{2} m_{s}^{2} \Phi_{S}^{2}+\frac{\lambda_{6}}{8} \Phi_{S}^{4}+\frac{\lambda_{7}}{2} \Phi_{1}^{\dagger} \Phi_{1} \Phi_{S}^{2}+\frac{\lambda_{8}}{2} \Phi_{2}^{\dagger} \Phi_{2} \Phi_{S}^{2},
\end{aligned}
$$

where all 11 free parameters of the Lagrangian,

$$
m_{11}^{2}, m_{22}^{2}, m_{S}^{2}, \lambda_{1-8}
$$

are real, or can be made to be so via a trivial rephasing of one of the doublets. Note that for the discrete symmetries to be exact we introduce no soft breaking terms in the potential. In particular, the term $m_{12}^{2}\left(\Phi_{1}^{\dagger} \Phi_{2}+\right.$ h.c. $)$ that would softly break the $Z^{(1)}$ symmetry is absent. This term is often used in many versions of the 2HDM and N2HDM to allow for a decoupling limit, with the introduction of the new mass scale $m_{12}^{2}$. After EWSB, the fields can be parametrised in terms of the charged complex fields $\phi_{i}^{+}(i \in\{1,2\})$, the neutral CP-even fields $\rho_{I}(I \in\{1,2, s\})$ and the neutral CP-odd fields $\eta_{i}$ as follows

$$
\Phi_{1}=\left(\begin{array}{c}
\phi_{1}^{+} \\
\frac{1}{\sqrt{2}}\left(v_{1}+\rho_{1}+i \eta_{1}\right)
\end{array}\right), \quad \Phi_{2}=\left(\begin{array}{c}
\phi_{2}^{+} \\
\frac{1}{\sqrt{2}}\left(v_{2}+\rho_{2}+i \eta_{2}\right)
\end{array}\right), \quad \Phi_{S}=v_{s}+\rho_{s} .
$$

Requiring the VEVs

$$
\left\langle\Phi_{i}\right\rangle=\left(\begin{array}{c}
0 \\
\frac{v_{i}}{\sqrt{2}}
\end{array}\right) \quad \text { and } \quad\left\langle\Phi_{S}\right\rangle=v_{s}
$$

which break the $\mathrm{SU}(2)_{L} \times \mathrm{U}(1)_{Y}$ down to $\mathrm{U}(1)_{E M}$, and possibly also the symmetries, to be solutions of the stationarity equations leads to the following three conditions,

$$
\begin{aligned}
& \left\langle\frac{\mathrm{d} V}{\mathrm{~d} v_{1}}\right\rangle=0 \Rightarrow-m_{11}^{2}=\frac{1}{2}\left(v_{1}^{2} \lambda_{1}+v_{2}^{2}\left(\lambda_{3}+\lambda_{4}+\lambda_{5}\right)+v_{s}^{2} \lambda_{7}\right), \\
& \left\langle\frac{\mathrm{d} V}{\mathrm{~d} v_{2}}\right\rangle=0 \Rightarrow-m_{22}^{2}=\frac{1}{2}\left(v_{1}^{2}\left(\lambda_{3}+\lambda_{4}+\lambda_{5}\right)+v_{2}^{2} \lambda_{2}+v_{s}^{2} \lambda_{8}\right), \\
& \left\langle\frac{\mathrm{d} V}{\mathrm{~d} v_{s}}\right\rangle=0 \Rightarrow-m_{s}^{2}=\frac{1}{2}\left(v_{1}^{2} \lambda_{7}+v_{2}^{2} \lambda_{8}+v_{s}^{2} \lambda_{6}\right) .
\end{aligned}
$$

If we consider only minima that are CP-conserving and non-charge breaking, we can distinguish four cases:

- The Broken Phase (BP) - In this phase both doublets and the singlet acquire VEVs and consequently both $\mathbb{Z}_{2}$ symmetries are spontaneously broken by EWSB. There are no dark matter candidates, and the scalar particle spectrum consists of three CPeven, one CP-odd and two charged scalars. This phase, with an extra soft breaking term for $\mathbb{Z}_{2}^{(1)}$, has been thoroughly studied in [17]. 
- The Dark Doublet Phase (DDP) - This is the case where only one of the doublets (either $\Phi_{1}$ or $\Phi_{2}$ ) and the singlet acquire VEVs. This phase is the N2HDM equivalent to the Inert Doublet Model of the $2 \mathrm{HDM}[10-13]$. The $\mathbb{Z}_{2}^{(1)}$ symmetry is exactly preserved while $\mathbb{Z}_{2}^{(2)}$ is spontaneously broken. There are four dark sector particles two neutral and a pair of charged scalars - and one extra CP-even scalar that mixes with the CP-even component from the doublet which acquires a VEV.

- The Dark Singlet Phase (DSP) - In this phase both doublets but not the singlet acquire VEVs. Hence, $\mathbb{Z}_{2}^{(2)}$ remains unbroken and the dark matter candidate has its origin in the singlet field. This phase is essentially a $2 \mathrm{HDM}$ plus a dark real singlet [7-9]. The model has two CP-even, one CP-odd and a pair of charged scalars in the visible sector plus a singlet-like DM particle.

- The Fully Dark Phase (FDP) - Finally, we will consider a phase where only one doublet acquires a VEV. This means that both $\mathbb{Z}_{2}$ symmetries remain unbroken and only one doublet couples to SM fields. Therefore, this model contains just one SMlike Higgs boson with additional couplings to dark particles. No new non-dark scalar is present and two distinct darkness quantum numbers are separately conserved.

We want the Lagrangian of the theory for all four phases to be exactly the same before EWSB. The kinetic terms are the same because they are only determined by the $\mathrm{SU}(2)_{L}$ and $\mathrm{U}(1)_{Y}$ quantum numbers. As for the Yukawa Lagrangian, the singlet field does not couple to the fermions and we have to choose a Yukawa sector of type I, where only one doublet couples to the fermions in order to be able to compare all four phases based on the same Lagrangian. The Yukawa Lagrangian takes the form,

$$
\mathcal{L}_{\text {Yukawa }}=-\bar{Q}_{L}^{T} Y_{U} \widetilde{\Phi}_{f} U_{R}-\bar{Q}_{L}^{T} Y_{D} \Phi_{f} D_{R}-\bar{L}_{L}^{T} Y_{L} \Phi_{f} E_{R}+\text { h.c. }
$$

where $\Phi_{f}$ is the doublet that couples to fermions, $Y$ are three-dimensional Yukawa coupling matrices in flavour space, the left-handed fermions are grouped into the doublets

$$
Q_{L}=\left(\begin{array}{c}
U_{L} \\
D_{L}
\end{array}\right)=\left(\begin{array}{c}
\left(u_{L}, c_{L}, t_{L}\right)^{T} \\
\left(d_{L}, s_{L}, b_{L}\right)^{T}
\end{array}\right), \quad L_{L}=\left(\begin{array}{c}
N_{L} \\
E_{L}
\end{array}\right)=\left(\begin{array}{c}
\left(\nu_{e, L}, \nu_{\mu, L}, \nu_{\tau, L}\right)^{T} \\
\left(e_{L}, \mu_{L}, \tau_{L}\right)^{T}
\end{array}\right)
$$

and the right-handed fermion into the singlets

$$
U_{R}=\left(u_{R}, c_{R}, t_{R}\right)^{T}, \quad D_{R}=\left(d_{R}, s_{R}, b_{R}\right)^{T}, \quad E_{R}=\left(e_{R}, \mu_{R}, \tau_{R}\right)^{T} .
$$

and $\widetilde{\Phi}_{f}$ stands for $\epsilon_{i j} \Phi_{f}^{*}$, with $\epsilon_{i j}$ given by

$$
\epsilon_{i j}=\left(\begin{array}{cc}
0 & 1 \\
-1 & 0
\end{array}\right)
$$

We will now describe the four phases in detail. 


\subsection{The broken phase (BP)}

In the broken phase, both the doublets and the singlet acquire VEVs that break both $\mathbb{Z}_{2}^{(1)}$ and $\mathbb{Z}_{2}^{(2)}$. Since the model was discussed in great detail in [17], we will just very briefly review the features of the model needed for this study.

The charged and pseudoscalar mass matrices are diagonalised via the rotation matrix

$$
R_{\beta}=\left(\begin{array}{cc}
c_{\beta} & s_{\beta} \\
-s_{\beta} & c_{\beta}
\end{array}\right)
$$

with $t_{\beta}=\frac{v_{2}}{v_{1}}$. Here and from now on we use the abbreviations $\sin x \equiv s_{x}, \cos x \equiv c_{x}$ and $\tan x \equiv t_{x}$. This yields the massless charged and neutral would-be Goldstone bosons $G^{ \pm}$and $G^{0}$, the charged Higgs mass eigenstates $H^{ \pm}$and the pseudoscalar mass eigenstate $A$. There are three CP-even gauge eigenstates $\left(\rho_{1}, \rho_{2}, \rho_{S}\right)$, two from the doublets and one from the singlet. The corresponding mass eigenstates $H_{1}, H_{2}$ and $H_{3}$, are obtained via the orthogonal mixing matrix $R$ parametrised as

$$
R=\left(\begin{array}{ccc}
c_{\alpha_{1}} c_{\alpha_{2}} & s_{\alpha_{1}} c_{\alpha_{2}} & s_{\alpha_{2}} \\
-\left(c_{\alpha_{1}} s_{\alpha_{2}} s_{\alpha_{3}}+s_{\alpha_{1}} c_{\alpha_{3}}\right) & c_{\alpha_{1}} c_{\alpha_{3}}-s_{\alpha_{1}} s_{\alpha_{2}} s_{\alpha_{3}} & c_{\alpha_{2}} s_{\alpha_{3}} \\
-c_{\alpha_{1}} s_{\alpha_{2}} c_{\alpha_{3}}+s_{\alpha_{1}} s_{\alpha_{3}} & -\left(c_{\alpha_{1}} s_{\alpha_{3}}+s_{\alpha_{1}} s_{\alpha_{2}} c_{\alpha_{3}}\right) & c_{\alpha_{2}} c_{\alpha_{3}}
\end{array}\right)
$$

in terms of the mixing angles $\alpha_{1}$ to $\alpha_{3}$, chosen to be in the range

$$
-\frac{\pi}{2} \leq \alpha_{1,2,3}<\frac{\pi}{2} .
$$

The matrix $R$ is defined is such a way that

$$
\left(\begin{array}{l}
H_{1} \\
H_{2} \\
H_{3}
\end{array}\right)=R\left(\begin{array}{c}
\rho_{1} \\
\rho_{2} \\
\rho_{S}
\end{array}\right)
$$

diagonalises the scalar mass matrix $M_{\text {scalar }}^{2}$,

$$
R M_{\text {scalar }}^{2} R^{T}=\operatorname{diag}\left(m_{H_{1}}^{2}, m_{H_{2}}^{2}, m_{H_{3}}^{2}\right) .
$$

We take, by convention,

$$
m_{H_{1}} \leq m_{H_{2}} \leq m_{H_{3}} .
$$

In the broken phase, the 11 parameters of the N2HDM, eq. (2.4), are expressed through the input parameters

$$
\alpha_{1}, \quad \alpha_{2}, \quad \alpha_{3}, \quad t_{\beta}, \quad v, \quad v_{S}, m_{H_{1,2,3}}, m_{A}, \quad m_{H^{ \pm}}
$$

The Higgs couplings $H_{i}(i=1,2,3)$ to the massive gauge bosons $V \equiv W, Z$ are written as

$$
i g_{\mu \nu} c\left(H_{i} V V\right) g_{H^{\mathrm{S} M} V V},
$$




\begin{tabular}{|cc|}
\hline \multicolumn{2}{|c|}{$c\left(H_{i} V V\right)$} \\
\hline$H_{1}$ & $c_{\alpha_{2}} c_{\beta-\alpha_{1}}$ \\
$H_{2}$ & $-c_{\beta-\alpha_{1}} s_{\alpha_{2}} s_{\alpha_{3}}+c_{\alpha_{3}} s_{\beta-\alpha_{1}}$ \\
$H_{3}$ & $-c_{\alpha_{3}} c_{\beta-\alpha_{1}} s_{\alpha_{2}}-s_{\alpha_{3}} s_{\beta-\alpha_{1}}$ \\
\hline
\end{tabular}

Table 1. The effective couplings $c\left(H_{i} V V\right)$ of the neutral CP-even N2HDM Higgs bosons $H_{i}$ to the massive gauge bosons $V=W, Z$.

\begin{tabular}{|cccc|}
\hline \multicolumn{4}{|c|}{ Type I } \\
\hline$c\left(H_{i} f f\right)$ & $u$ & $d$ & $l$ \\
\hline$H_{1}$ & $\left(c_{\alpha_{2}} s_{\alpha_{1}}\right) / s_{\beta}$ & $\left(c_{\alpha_{2}} s_{\alpha_{1}}\right) / s_{\beta}$ & $\left(c_{\alpha_{2}} s_{\alpha_{1}}\right) / s_{\beta}$ \\
$H_{2}$ & $\left(c_{\alpha_{1}} c_{\alpha_{3}}-s_{\alpha_{1}} s_{\alpha_{2}} s_{\alpha_{3}}\right) / s_{\beta}$ & $\left(c_{\alpha_{1}} c_{\alpha_{3}}-s_{\alpha_{1}} s_{\alpha_{2}} s_{\alpha_{3}}\right) / s_{\beta}$ & $\left(c_{\alpha_{1}} c_{\alpha_{3}}-s_{\alpha_{1}} s_{\alpha_{2}} s_{\alpha_{3}}\right) / s_{\beta}$ \\
$H_{3}$ & $-\left(c_{\alpha_{1}} s_{\alpha_{3}}+c_{\alpha_{3}} s_{\alpha_{1}} s_{\alpha_{2}}\right) / s_{\beta}$ & $-\left(c_{\alpha_{1}} s_{\alpha_{3}}+c_{\alpha_{3}} s_{\alpha_{1}} s_{\alpha_{2}}\right) / s_{\beta}$ & $-\left(c_{\alpha_{1}} s_{\alpha_{3}}+c_{\alpha_{3}} s_{\alpha_{1}} s_{\alpha_{2}}\right) / s_{\beta}$ \\
\hline
\end{tabular}

Table 2. The effective Yukawa couplings $c\left(H_{i} f f\right)$ of the N2HDM Higgs bosons $H_{i}$, as defined in eq. (2.20) for Type I.

where $g_{H S M V V}$ is the SM Higgs coupling to the massive gauge bosons, and the coupling modifiers $c\left(H_{i} V V\right)$ are presented in table 1 . As previously discussed the four phases of the N2HDM can only be compared for the Yukawa Type I. The Yukawa Lagrangian reads

$$
\mathcal{L}_{Y}=-\sum_{i=1}^{3} \frac{m_{f}}{v} c\left(H_{i} f f\right) \bar{\psi}_{f} \psi_{f} H_{i}
$$

where the effective coupling factors $c\left(H_{i} f f\right)$ are shown in table 2. The remaining couplings are discussed in [17].

\subsection{The dark doublet phase (DDP)}

In the DDP only one of the two doublets and the singlet acquire VEVs and the $\mathbb{Z}_{2}^{(1)}$ symmetry forces all the fields in the other doublet to conserve the darkness parity. The lightest of these dark scalars is a DM candidate.

Assuming that $\Phi_{1}$ is the SM-like doublet, the vacuum configuration in the DDP is given by

$$
\left\langle\Phi_{1}\right\rangle=\frac{1}{\sqrt{2}}\left(\begin{array}{l}
0 \\
v
\end{array}\right), \quad\left\langle\Phi_{2}\right\rangle=\left(\begin{array}{l}
0 \\
0
\end{array}\right), \quad\left\langle\Phi_{S}\right\rangle=v_{s},
$$

where $v \approx 246 \mathrm{GeV}$ is the electroweak VEV and $v_{s} \neq 0$ is the singlet VEV. The difference between the non-dark sector and the SM is that the singlet $\rho_{s}$ will mix with the CP-even $\rho_{1}$. The mass eigenstates $H_{i}(i=1,2,3)$ are obtained from $\left(\rho_{1}, \rho_{2}, \rho_{S}\right)$ via the rotation matrix

$$
\mathcal{R}=\left(\begin{array}{ccc}
\cos \alpha & 0 & \sin \alpha \\
-\sin \alpha & 0 & \cos \alpha \\
0 & 1 & 0
\end{array}\right)
$$


By convention, we order the visible $H_{i}$ by ascending mass

$$
m_{H_{1}} \leq m_{H_{2}}
$$

and choose the third mass eigenstate $H_{D} \equiv H_{3}=\rho_{S}$. There is no mixing between the remaining components of the two doublets and therefore

$$
\begin{aligned}
G^{0} & =\eta_{1}, & & A_{D}=\eta_{2}, \\
G^{ \pm} & =\phi_{1}^{ \pm}, & & H_{D}^{ \pm}=\phi_{2}^{ \pm} .
\end{aligned}
$$

The Goldstone bosons are in the SM-like doublet and the dark charged and dark CP-odd particles are in the inert doublet. ${ }^{1}$

In the DDP, the 11 parameters of the N2HDM, eq. (2.4), are expressed through

$$
v, v_{s}, m_{H_{1}}, m_{H_{2}}, m_{H_{D}}, m_{A_{D}}, m_{H_{D}^{ \pm}}, \alpha, m_{22}^{2}, \lambda_{2}, \lambda_{8} .
$$

The explicit parameter transformations are given in appendix A.

The couplings of the scalars to the remaining SM particles can be grouped into a visible sector consisting of the two neutral CP-even fields $H_{1}$ and $H_{2}$ and the dark sector with the four scalars $H_{D}, A_{D}$ and $H_{D}^{ \pm}$. The coupling modifiers in the visible sector are given by

$$
c\left(H_{i}(p)\right)=\frac{\lambda_{i}^{(p)}}{\lambda_{S M}^{(p)}}=\mathcal{R}_{i 1}
$$

where $H_{i}(i=1,2)$ and $p$ stands for a pair of SM particles, provided that there is a corresponding coupling in the SM. $\lambda$ stands for the Feynman rule of the corresponding vertex and the division by $\lambda_{S M}$ is taken to cancel identical tensor structures. Because this visible sector is just the extension of the SM by a real singlet the following sum rules hold:

$$
\sum_{i=1}^{2} c^{2}\left(H_{i} \bar{f} f\right)=\sum_{i=1}^{2} c^{2}\left(H_{i} V V\right)=1 .
$$

Finally no FCNC occur at tree-level because only the first doublet couples to fermions.

Due to the unbroken $\mathbb{Z}_{2}^{(1)}$ symmetry the dark scalars $H_{D}^{ \pm}, H_{D}$ and $A_{D}$ do not couple to either pairs of fermion or pairs of gauge bosons. However - because of the doublet nature of $\Phi_{2}$ - there are couplings involving two dark scalars and one vector boson in addition to the triple-Higgs couplings $H_{i} H_{D} H_{D}, H_{i} A_{D} A_{D}$ and $H_{i} H_{D}^{ \pm} H_{D}^{\mp}$ that link the dark and the visible sectors. The trilinear Higgs gauge couplings are dependent on the momenta of the scalars and there is no SM equivalent with which they could be normalised. Adopting the convention in which the momentum $p_{H_{D}}$ of $H_{D}$ is incoming, and the momenta $p_{A_{D}}$ and

\footnotetext{
${ }^{1}$ Note that just like in the IDM there is no way to tell which of $H_{D}$ and $A_{D}$ is the CP-even and which is the CP-odd state. In fact, since both $H_{D}$ and $A_{D}$ do not couple to fermions, it is just the $H_{D} A_{D} Z$ coupling that tells us they have opposite CP. Regardless, we will call $H_{D} \mathrm{CP}$-even and $A_{D} \mathrm{CP}$-odd throughout this paper for simplicity.
} 
$p_{H_{D}^{ \pm}}$of the scalars $A_{D}$ or $H_{D}^{ \pm}$are outgoing, we get the following Feynman rules

$$
\begin{aligned}
\lambda^{\mu}\left(H_{D}, A_{D}, Z\right) & =-\frac{\sqrt{g^{2}+g^{\prime 2}}}{2}\left(p_{A_{D}}+p_{H_{D}}\right)^{\mu}, \\
\lambda^{\mu}\left(H_{D}, H_{D}^{ \pm}, W^{\mp}\right) & =\mp \frac{i g}{2}\left(p_{H_{D}^{ \pm}}+p_{H_{D}}\right)^{\mu} .
\end{aligned}
$$

These, and the Feynman rules for the vertices $A_{D} H_{D}^{ \pm} W^{\mp}, H_{D}^{ \pm} H_{D}^{\mp} Z$ and $H_{D}^{ \pm} H_{D}^{\mp} \gamma$ are the same as in the $2 \mathrm{HDM}$ and can be found in ref. [19]. The triple Higgs couplings are given in appendix A.

\subsection{The dark singlet phase (DSP)}

In the DSP only the doublets acquire VEVs which means that the $\mathbb{Z}_{2}^{(2)}$ symmetry is left unbroken. In turn, only the CP-even fields $\rho_{1}$ and $\rho_{2}$ mix and $\rho_{S}$ is the DM candidate. Now, the vacuum configuration is

$$
\left\langle\Phi_{1}\right\rangle=\frac{1}{\sqrt{2}}\left(\begin{array}{c}
0 \\
v_{1}
\end{array}\right), \quad\left\langle\Phi_{2}\right\rangle=\frac{1}{\sqrt{2}}\left(\begin{array}{c}
0 \\
v_{2}
\end{array}\right), \quad\left\langle\Phi_{S}\right\rangle=0,
$$

where $v_{1}=v \cos \beta$ and $v_{2}=v \sin \beta$ and $v$ is the electroweak VEV. To rotate from the gauge eigenstates $\left(\rho_{1}, \rho_{2}, \rho_{S}\right)$ to the mass eigenstates we define a rotation matrix compatible with the usual $2 \mathrm{HDM}$ definition,

$$
\mathcal{R}=\left(\begin{array}{ccc}
-\sin \alpha & \cos \alpha & 0 \\
\cos \alpha & \sin \alpha & 0 \\
0 & 0 & 1
\end{array}\right)
$$

where we use the mass ordering

$$
m_{H_{1}} \leq m_{H_{2}} .
$$

$H_{3}=\rho_{S}$ is the dark scalar $H_{D}$. The CP-odd and charged eigenstates are obtained exactly like in the $2 \mathrm{HDM}$ case, that is,

$$
\begin{aligned}
G^{0} & =\eta_{1} \cos \beta+\eta_{2} \sin \beta, & A & =-\eta_{1} \sin \beta+\eta_{2} \cos \beta, \\
G^{ \pm} & =\phi_{1}^{ \pm} \cos \beta+\phi_{2}^{ \pm} \sin \beta, & H^{ \pm} & =-\phi_{1}^{ \pm} \sin \beta+\phi_{2}^{ \pm} \cos \beta .
\end{aligned}
$$

In the DSP, the 11 parameters of the N2HDM, eq. (2.4), are expressed in terms of the input parameters as

$$
v, \tan \beta, m_{H_{1}}, m_{H_{2}}, m_{H_{D}}, m_{A}, m_{H^{ \pm}}, \alpha, \lambda_{6}, \lambda_{7}, \lambda_{8},
$$

and the explicit transformation of the parameters can be found in appendix B.

Regarding the Higgs couplings, the singlet field $\rho_{S}$ does not couple to SM particles nor does it mix with the remaining CP-even scalar fields $\rho_{1}$ and $\rho_{2}$. Hence the $H_{1}$ and $H_{2}$ couplings to the SM particles are just the 2HDM Type I ones and can be found in table 3. 


\begin{tabular}{|rcr|}
\hline & $c\left(H_{i} \bar{f} f\right)$ & $c\left(H_{i} V V\right)$ \\
\hline$H_{1}$ & $\cos \alpha / \sin \beta$ & $-\sin (\alpha-\beta)$ \\
$H_{2}$ & $\sin \alpha / \sin \beta$ & $\cos (\alpha-\beta)$ \\
\hline
\end{tabular}

Table 3. Yukawa and gauge boson coupling modifiers for the CP-even Higgs bosons $H_{i}(i=1,2)$ in the DSP.

The only additional couplings are the triple-Higgs couplings $H_{i} H_{D} H_{D}(i=1,2)$, which allow for the decay of the light and heavy CP-even Higgs boson into DM if kinematically possible. These interactions have the form

$$
g\left(H_{i} H_{D} H_{D}\right)=\frac{\partial \mathcal{L}}{\partial H_{i} \partial H_{D} \partial H_{D}}=\lambda_{7} v \cos \beta \mathcal{R}_{i 1}+\lambda_{8} v \sin \beta \mathcal{R}_{i 2},
$$

where $\mathcal{R}_{i j}$ is the $i j$ element of the mixing matrix in eq. (2.32).

\subsection{The fully dark phase (FDP)}

In the FDP only one doublet acquires a VEV. This means that both $\mathbb{Z}_{2}^{(1)}$ and $\mathbb{Z}_{2}^{(2)}$ remain unbroken and we have two DM candidates corresponding to the two different dark parities. Because all other neutral fields belong to one of the dark phases, the SM-like Higgs is just the one from the doublet with a VEV. There is no mixing in the scalar sector, such that $\mathcal{R}=\mathbb{1}_{3 \times 3}$ in the basis

$$
\left(\begin{array}{c}
H_{\mathrm{SM}} \\
H_{D}^{D} \\
H_{D}^{S}
\end{array}\right)=R\left(\begin{array}{c}
\rho_{1} \\
\rho_{2} \\
\rho_{S}
\end{array}\right)
$$

where we denote by $H_{D}^{D}\left(H_{D}^{S}\right)$ the CP-even, dark scalar from the doublet (singlet). Hence, $H_{\mathrm{SM}}$ has exactly the same couplings to SM particles as in the SM. The only difference relative to the SM are the couplings between the Higgs and the dark matter candidates stemming from the Higgs potential. There is, however, a difference in the SM Higgs radiative decays and in particular $H_{\mathrm{SM}} \rightarrow \gamma \gamma$ where the contribution from the dark charged Higgs loops can significantly change $\Gamma(H \rightarrow \gamma \gamma)$. In the FDP, the 11 parameters of the N2HDM, eq. (2.4), are expressed through

$$
v, m_{H_{S M}}, m_{H_{D}^{D}}, m_{H_{D}^{S}}, m_{A_{D}}, m_{H_{D}^{ \pm}}, m_{22}^{2}, m_{S}^{2}, \lambda_{2}, \lambda_{6}, \lambda_{8} .
$$

\section{Neutral vacua stability}

The existence of several possible vacua, wherein different discrete symmetries of the model are broken by the vevs, raises the possibility of coexisting minima. Namely, is it guaranteed that once we find a given minimum - corresponding to one of the phases defined in section 2 - that this minimum is the global one? Or may deeper neutral minima exist, raising the possibility of tunnelling between minima? In order to answer this question one must compute the values of the potential at different coexisting vacua and compare them. In the context of charge breaking vacua in the N2HDM the authors of the present work 


\begin{tabular}{|lc|}
\hline Phase & vevs \\
\hline BP & $v_{1}^{B}, v_{2}^{B}, v_{s}^{B}$ \\
DDP & $v_{1}^{D}, v_{s}^{D}$ \\
DSP & $v_{1}^{S}, v_{2}^{S}$ \\
FDP & $v_{1}^{F}$ \\
\hline
\end{tabular}

Table 4. Naming convention for the vevs at stationary points of different phases. Only non-zero vevs are shown.

analysed this possibility in ref. [20] (see also [21, 22]). We now undertake a similar study for coexisting neutral vacua following earlier numerical studies in refs. [17, 23].

To begin with, some generic considerations:

- In all that follows, we will always assume that two stationary points, corresponding to different phases of the model, coexist. This means that, for some set of parameters of the potential, we are assuming that the minimization conditions of the potential admit two solutions, with different values for the vevs.

- Since we will be comparing the values of the potential at different phases of the model, we must distinguish between the vevs $v_{1}, v_{2}$ and $v_{s}$ defined previously. Therefore, each vev will, for the purposes of this section alone, be tagged with a superscript to specify which neutral phase is being discussed. The vevs of the Broken Phase (BP), for instance, will be tagged with a "B" $-v_{1}^{B}, v_{2}^{B}$ and $v_{s}^{B}$ - whereas those of the Dark Doublet Phase (DDP) will carry a "D" $-v_{1}^{D}$ and $v_{s}^{D}$. The complete correspondence can be found in table 4. Likewise, scalar masses at different phases will carry the same subscript

In order to compare the values of the potential at different vacua we will deploy a bilinear formalism similar to the one employed for the 2HDM [24-37]. In this approach, bilinears are several gauge-invariant quantities, quadratic in the fields, and the potential, expressed in terms of these variables, becomes a quadratic polynomial. The minimisation of the potential is greatly simplified, and geometrical properties of these bilinears permit a detailed analysis of symmetries of the potential and its vacuum structure. This formalism has been adapted to study the vacuum structure of other models, such as the three-Higgs doublet model [38-40], the doublet-singlet model [41], the N2HDM [20] and the Higgstriplet model [42]. We now give a brief overview of the technique: let us define vectors $A$ and $X$ and a matrix $B$ as

$$
X=\frac{1}{2}\left(\begin{array}{c}
v_{1}^{2} \\
v_{2}^{2} \\
v_{1} v_{2} \\
v_{s}^{2}
\end{array}\right), \quad A=\left(\begin{array}{c}
m_{11}^{2} \\
m_{22}^{2} \\
0 \\
m_{S}^{2}
\end{array}\right), \quad B=\left(\begin{array}{cccc}
\lambda_{1} & \lambda_{3} & 0 & \lambda_{7} \\
\lambda_{3} & \lambda_{2} & 0 & \lambda_{8} \\
0 & 0 & 2\left(\lambda_{4}+\lambda_{5}\right) & 0 \\
\lambda_{7} & \lambda_{8} & 0 & \lambda_{6}
\end{array}\right)
$$


The value of the potential of eq. (2.3) at any of the phases (corresponding to a stationary point (SP)) we consider in this work can be then be expressed as

$$
V_{S P}=A^{T} X_{S P}+\frac{1}{2} X_{S P}^{T} B X_{S P},
$$

with the vector $X$ evaluated at the stationary point, and it can easily be shown that, due to the minimisation conditions, one has

$$
V_{S P}=\frac{1}{2} A^{T} X_{S P}=-\frac{1}{2} X_{S P}^{T} B X_{S P} .
$$

The bilinear formalism also requires that we define the following vector

$$
V_{S P}^{\prime}=\frac{\partial V}{\partial X^{T}}=A+B X_{S P} .
$$

In order to illustrate the technique we will now show how to apply the formalism to one of the cases we are interested in, detailing the several steps needed to reach a formula comparing the depth of the potential at two different phases. We will then simply present the results obtained for all the other cases without demonstration.

Suppose the N2HDM potential of eq. (2.3) has two stationary points, corresponding to the phases $\mathrm{BP}$ and DDP, defined in section 2. Then, the vectors $X$ and $V^{\prime}$ have the following expressions for each phase: for the Broken Phase,

$$
X_{B P}=\frac{1}{2}\left(\begin{array}{c}
\left(v_{1}^{B}\right)^{2} \\
\left(v_{2}^{B}\right)^{2} \\
v_{1}^{B} v_{2}^{B} \\
\left(v_{s}^{B}\right)^{2}
\end{array}\right), \quad V_{B P}^{\prime}=A+B X_{B P}=-\frac{\lambda_{4}+\lambda_{5}}{2}\left(\begin{array}{c}
\left(v_{2}^{B}\right)^{2} \\
\left(v_{1}^{B}\right)^{2} \\
-2 v_{1}^{B} v_{2}^{B} \\
0
\end{array}\right)
$$

and for the Dark Doublet Phase,

$$
X_{D D P}=\frac{1}{2}\left(\begin{array}{c}
\left(v_{1}^{D}\right)^{2} \\
0 \\
0 \\
\left(v_{s}^{D}\right)^{2}
\end{array}\right), \quad V_{D D P}^{\prime}=A+B X_{D D P}=-\left(m_{H^{ \pm}}^{2}\right)^{D}\left(\begin{array}{l}
0 \\
1 \\
0 \\
0
\end{array}\right),
$$

where the charged scalar mass at the DDP extremum is given by

$$
\left(m_{H^{ \pm}}^{2}\right)^{D}=m_{22}^{2}+\frac{1}{2} \lambda_{3}\left(v_{1}^{D}\right)^{2}+\frac{1}{2} \lambda_{8}\left(v_{s}^{D}\right)^{2} .
$$

We then compute the following product between vectors:

$$
\begin{aligned}
X_{B P}^{T} V_{D D P}^{\prime} & =X_{B P}^{T} A+X_{B P}^{T} B X_{D D P}=2 V_{B P}+X_{B P}^{T} B X_{D D P} \\
& =-\frac{\lambda_{4}+\lambda_{5}}{4}\left(v_{2}^{B}\right)^{2}\left(v_{1}^{D}\right)^{2}
\end{aligned}
$$

where in the second line we used the result from eq. (3.3). Likewise, we obtain

$$
\begin{aligned}
X_{D D P}^{T} V_{B P}^{\prime} & =X_{D D P}^{T} A+X_{D D P}^{T} B X_{B P}=2 V_{D D P}+X_{D D P}^{T} B X_{B P} \\
& =\frac{1}{2}\left(v_{2}^{B}\right)^{2}\left(m_{H^{ \pm}}^{2}\right)^{D} .
\end{aligned}
$$


Since the matrix $B$ is symmetric we will have $X_{B P}^{T} B X_{D D P}=X_{D D P}^{T} B X_{B P}$, and therefore, subtracting the two equations above one from another we obtain, after some intermediate steps that we skip for brevity,

$$
V_{B P}-V_{D D P}=\frac{1}{4}\left(v_{2}^{B}\right)^{2}\left(m_{H_{D}}^{2}\right)^{D},
$$

where $\left(m_{H_{D}}^{2}\right)^{D}$ is the squared scalar mass corresponding of the real, neutral component of the doublet $\Phi_{2}$ in the DDP phase (see appendix A). What eq. (3.10) shows us is that, if the Dark Doublet Phase is a minimum, then all of the squared scalar masses therein computed will perforce be positive and then one will necessarily have

$$
V_{B P}-V_{D D P}>0 \quad \text { if DDP is a minimum. }
$$

Therefore, if DDP is a minimum, any stationary point corresponding to the Broken Phase will necessarily lie above that minimum.

Following similar steps we can obtain the relations between the BP potential value and the remaining phases, namely

$$
\begin{aligned}
& V_{B P}-V_{D S P}=\frac{1}{4}\left(v_{s}^{B}\right)^{2}\left(m_{H_{D}}^{2}\right)^{S}, \\
& V_{B P}-V_{F D P}=\frac{1}{4}\left(v_{2}^{B}\right)^{2}\left(m_{H_{D}^{D}}^{2}\right)^{F}+\frac{1}{4}\left(v_{s}^{B}\right)^{2}\left(m_{H_{D}^{S}}^{2}\right)^{F},
\end{aligned}
$$

where the $m^{2}$ are physical scalar masses at the given phases. From these equations one draws analogous conclusions to the case with coexisting BP and DDP phases.

The above does not answer the question of whether a local BP minimum could coexist with a deeper DDP, DSP or FDP minimum, however. We will now show that any BP stationary point will necessarily be a saddle point: in the BP phase, the real neutral components of both doublets, $\rho_{1}$ and $\rho_{2}$, mix with the singlet component field $\rho_{S}$, leading to a $3 \times 3$ scalar mass matrix for the CP-even scalars, $M_{\text {scalar }}^{2}$ (see section 2). It is possible to show that there is an alternative way of writing eq. (3.10), to wit

$$
V_{B P}-V_{D D P}=\frac{1}{4}\left(v_{2}^{B}\right)^{2}\left(m_{H_{D}}^{2}\right)^{D}=-\frac{1}{8\left(\lambda_{1} \lambda_{6}-\lambda_{7}^{2}\right)} \frac{\left(v_{2}^{B}\right)^{2}}{\left(v_{1}^{B}\right)^{2}\left(v_{s}^{B}\right)^{2}} \operatorname{det}\left(M_{\text {scalar }}^{2}\right)_{B},
$$

where we added the subscript " $\mathrm{B}$ " to the determinant to emphasise that these scalar masses are evaluated at the BP extremum. It can be shown that, if the DDP phase is a minimum, then one must have $\lambda_{1} \lambda_{6}-\lambda_{7}^{2}>0$ (to do this one must look at the DDP scalar mass matrix, see appendix A). Therefore, if the DDP is a minimum then $V_{B P}-V_{D D P}>0$ and $\operatorname{det}\left(M_{\text {scalar }}^{2}\right)_{B}<0$ - which means that at least one BP squared scalar mass is negative. Since $\left(M_{\text {scalar }}^{2}\right)_{B}$ is a matrix with positive diagonal entries some of its minors are guaranteed to be positive - and therefore we conclude that at least one of its eigenvalues is positive. Therefore, if the DDP is a minimum, the broken phase BP is a saddle point. Reversely, if the $\mathrm{BP}$ is a minimum, then one will have $V_{B P}-V_{D D P}<0$ and the DDP extremum cannot be a minimum, and indeed it can be shown to be a saddle point. Analogous expressions can be found for the comparison between the BP and the other neutral phases. Thus one may conclude the following: 
- If any of the phases DDP, DSP and FDP is a minimum, then any stationary point of the BP lies necessarily above that minimum, and is a saddle point.

- If there is a minimum of the scalar potential in the BP, then any stationary points of the DDP, DSP and FDP are necessarily saddle points and lie above the BP minimum.

We can easily find the relationship between the depths of the potential at DDP and DSP phases - analogous calculations lead us to

$$
V_{D S P}-V_{D D P}=\frac{1}{4}\left(v_{2}^{S}\right)^{2}\left(m_{H_{D}}^{2}\right)^{D}-\frac{1}{4}\left(v_{s}^{D}\right)^{2}\left(m_{H_{D}}^{2}\right)^{S},
$$

where we see that now, even if either the DSP or the DDP, or both, are minima, there is no assurance whatsoever that it is the deepest minimum. In fact, the above expression, from previous $2 \mathrm{HDM}$ and N2HDM experience, implies that DSP and DDP minima can coexist and either can be the deepest minimum, depending on the choice of parameters of the potential.

Finally, one can analyse the FDP phase. We already saw (eq. (3.13) above) that an FDP minimum implies that any BP extrema lies above it. When we compare FDP stationary points with DDP and DSP ones, we obtain the following expressions,

$$
\begin{aligned}
& V_{D D P}-V_{F D P}=\frac{1}{4}\left(v_{s}^{D}\right)^{2}\left(m_{H_{D}^{S}}^{2}\right)^{F}, \\
& V_{D S P}-V_{F D P}=\frac{1}{4}\left(v_{2}^{S}\right)^{2}\left(m_{H_{D}^{D}}^{2}\right)^{F},
\end{aligned}
$$

which again show that, if the FDP is a minimum, any extrema corresponding to the phases DDP and DSP necessarily will lie above it - and as happened for the BP phase, it can be shown that in that case the DDP and DSP phases would not be minima, but rather saddle points. Likewise, the existence of DDP/DSP minima would imply that any FDP extremum would lie above it, and it would be a saddle point. From eq. (3.13) and these results we can therefore safely conclude that a minimum in the FDP is deeper than any other extrema for different neutral phases.

To summarise, then:

- If a BP minimum exists it is the global minimum of the theory. All other stationary points corresponding to different phases lie above it and are saddle points.

- Likewise for the existence of a FDP minimum - if it exists it is global and all other stationary points corresponding to different phases lie above it and are saddle points.

- However, minima of the DDP and DSP can coexist in the potential, and neither is guaranteed to be deeper than the other. If there is a minimum DDP or DSP, any BP or FDP extrema are saddle points above it.

This last point recalls the coexistence of minima which break the same symmetries in the 2HDM [33]. Although in the DDP and DSP phases different symmetries are broken, the symmetry of these models after spontaneous symmetry breaking is very similar in both models, as a $\mathbb{Z}_{2}$ symmetry is left unbroken by the vacuum in both models. 
We therefore were able to find general statements about the N2HDM vacuum structure in an analytical manner. Stability of BP and FDP phases is assured, but numerical checks need to be performed on DDP and DSP ones in order to verify whether a minimum of these phases is the global one. A final note about having set $m_{12}^{2}=0$. As already discussed in [20], if $m_{12}^{2} \neq 0$ the result that compares the BP with the DSP no longer holds. Let us now proceed to the numerical analysis of the several phases.

\section{Parameter scans and constraints}

All phases of the N2HDM have been implemented in the ScannerS code [43, 44] to perform parameter scans and in the N2HDECAY code $[17,45]$ to calculate all Higgs branching ratios and decay widths including state-of-the-art higher-order QCD corrections and off-shell decays. Electroweak corrections, which - in contrast to the QCD corrections - cannot be taken over from the SM, have been consistently neglected. ${ }^{2}$ Since we only consider type I Yukawa sectors - where the effective couplings of each visible Higgs boson to all fermions are equal - the scalar production cross sections are easily obtained for all phases from the corresponding SM ones - calculated using SusHi v1.6.1 [47, 48] (see also [49]).

The parameter points generated using ScannerS in each model are in agreement with the most relevant theoretical and experimental constraints. Theoretical constraints include that the potential is bounded from below and that perturbative unitarity holds [17]. We further require stability of the EW vaccuum, and also allow for metastability using the numerical procedure described in refs. $[20,50]$, provided the tunnelling time to a deeper minimum is larger than the age of the Universe. The SM-like Higgs boson mass is taken to be [51]

$$
m_{h_{125}}=125.09 \mathrm{GeV},
$$

and to preclude interference with other Higgs signals we force any non-dark neutral scalar to be outside the $m_{h_{125}} \pm 5 \mathrm{GeV}$ mass window. Any of the visible CP-even Higgs bosons can be the discovered one.

Compatibility with electroweak precision data is imposed by a 95\% C.L. exclusion limit from the electroweak precision observables $S, T$ and $U$ [52] using the formulae in refs. $[53,54]$ and the fit result of ref. [55]. In the BP and DSP we also consider constraints from charged-Higgs mediated contributions to $b$-physics observables [55].

Constraints from Higgs searches are taken into account using the combined 95\% C.L. exclusion bound constructed by HiggsBounds-5.7.1 [56-58] including LEP, Tevatron and LHC results. The measurements of the $h_{125}$ properties at the LHC are included through the use of HiggsSignals-2.4.0 [59], where a $\Delta \chi^{2}<6.18$ cut relative to the SM is used.

In the dark phases, additional constraints from DM observables are considered. The relic density and direct detection cross sections are calculated using MicrOMEGAs5.0.9 [60-66]. This calculation correctly accounts for the two-component DM in the FDP.

\footnotetext{
${ }^{2}$ While there exists a the code ewN2HDECAY [46] that calculates the electroweak corrections to the onshell and not loop-induced decays of the neutral N2HDM Higgs bosons in the broken phase it has not been adapted yet to the dark phases discussed in this paper.
} 

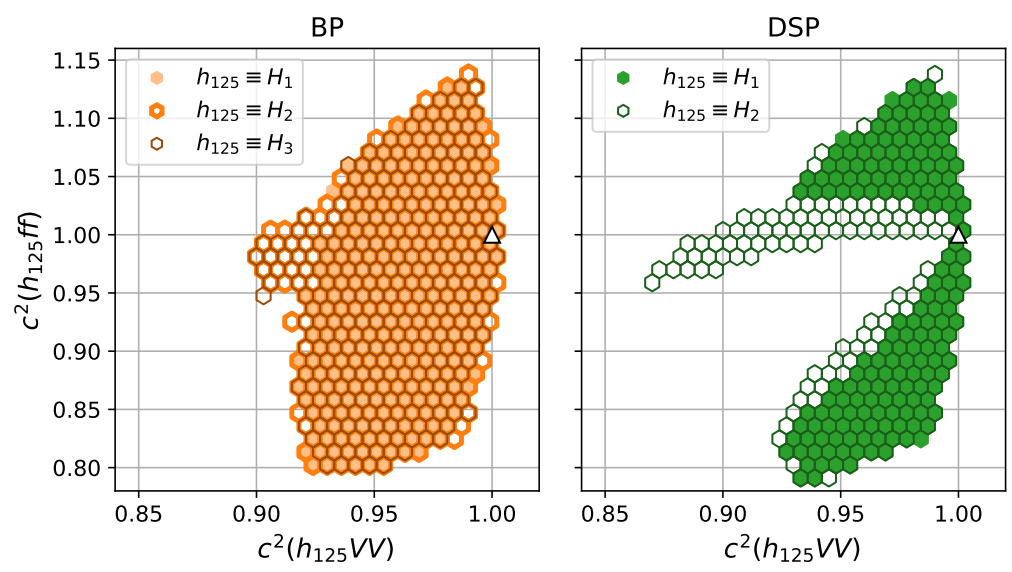

Figure 1. Coupling modifiers relative to the SM for the couplings of $h_{125}$ to fermions, $c\left(h_{125} \bar{f} f\right)$, and to gauge bosons, $c\left(h_{125} V V\right)$, for the Broken Phase (left) and for the Dark Singlet Phase (right). The white triangle indicates the SM value.

The model-predicted relic density is required not to oversaturate the observed relic abundance [67] by more than $2 \sigma$. Additionally, the direct detection bound by the Xenon1t experiment [68] is imposed.

Let us now understand what are the present bounds on the Higgs couplings modifiers. In figure 1 we present the squared coupling modifiers to fermions and to gauge bosons of the $125 \mathrm{GeV}$ Higgs boson $h_{125}$. We show the Broken Phase (left) and the Dark Singlet Phase (right). Due to unitarity, the effective coupling to gauge bosons cannot exceed 1. We also show the differences in the allowed parameter space when considering the different CP-even scalars as the $h_{125}$. In both phases, we see that lower values of $c^{2}\left(h_{125} V V\right)$ are allowed if $h_{125}$ is not the lightest of the $H_{i}$. This is the result of more freedom in $\mu_{\gamma \gamma}$ for light spectra - in particular for light charged Higgs masses. We will explain the origin of this behaviour below, when we discuss $\mu_{\gamma \gamma}$ as a distinguishing factor between the phases. We do not show the corresponding plots for the other two phases since they are trivial. In the DDP the two effective couplings are always equal and constrained to the experimentally allowed range

$$
0.87<c^{2}\left(h_{125} f f\right)=c^{2}\left(h_{125} V V\right)<1,
$$

while in the FDP, both couplings take exactly their SM values.

In figure 2 we show the branching ratio of $h_{125}$ to DM particles vs. the quantity

$$
\mu_{V V}=\frac{\sigma\left(p p \rightarrow h_{125} \rightarrow Z Z\right)}{\sigma_{\mathrm{SM}}\left(p p \rightarrow h_{125} \rightarrow Z Z\right)}=\frac{\sigma\left(p p \rightarrow h_{125} \rightarrow W^{+} W^{-}\right)}{\sigma_{\mathrm{SM}}\left(p p \rightarrow h_{125} \rightarrow W^{+} W^{-}\right)},
$$

for the three dark phases. The maximum allowed value of the branching ratio of the Higgs decaying to DM particles is below $10 \%$ in all phases. The present experimental bound on $\mathrm{BR}\left(h_{125} \rightarrow\right.$ invisible $)$ is about $26 \%$ [69]. This means that indirect constraints on $\mathrm{BR}\left(h_{125} \rightarrow\right.$ invisible $)$ from the Higgs rate measurements are significantly stronger than those from direct searches for invisible decays of $h_{125}$. 


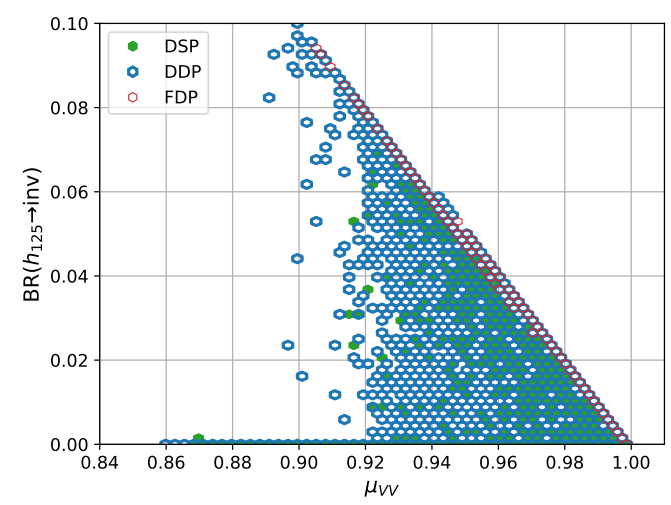

Figure 2. Branching ratio of $h_{125}$ to DM particles vs. $\mu_{V V}$ for the three DM phases.

Let us now move to the DM constraints. The analysis of the DM phases are the main goal of this study. Therefore, we need to make sure that the DM candidates are compatible with the corresponding experimental constraints. The Planck space telescope [67] maps the anisotropies in the cosmic microwave background radiation. We force our points to have a relic density of cold dark matter within or below the $2 \times 1 \sigma$ band of the experimental fit value

$$
\left(\Omega_{c} h^{2}\right)_{\exp }=0.1200 \pm 0.0012 .
$$

Hence, points with an over-abundance of DM are excluded. These models are also constrained by DM direct detection. The most recent results are the ones from the XENON1T experiment [70] a dual phase (liquid-gas) Xenon time projection chamber. Because no signal has been observed so far, constraints in the plane DM-nucleon cross section vs. DM mass are obtained. Since the XENON1T bound is obtained assuming a relic density equal to eq. (4.4) and we allow for smaller values of the relic densities, the impact of the DM abundance on direct detection measurements is taken into account by considering a normalised scattering cross section $\hat{\sigma}_{D M-N}$, given by

$$
\hat{\sigma}_{\mathrm{DM}-\mathrm{N}}=\sigma_{\mathrm{DM}-\mathrm{N}} \frac{\Omega_{c} h^{2}}{\left(\Omega_{c} h^{2}\right)_{\exp }},
$$

where $\sigma_{\mathrm{DM}-\mathrm{N}}$ and $\Omega_{c} h^{2}$ are the values calculated for a given parameter set.

In figure 3 we present the Nucleon-DM cross section, $\hat{\sigma}_{\mathrm{DM}-\mathrm{N}}$, as a function of the DM mass with all the constraints previously discussed. The colour code represents the fraction of the DM relic density where the upper limit is the central value measured by Planck plus $2 \times 1 \sigma$. Regarding direct detection it is clear that plenty of parameter points will survive all the way down to the neutrino floor [71] — which for the mass range in question is of the order of $10^{-12} \mathrm{pb}$. As for saturating the relic density - allowing therefore that DM is fully explained within the model - we now refer to figure 4 for clarity. In the figure we see that except for the DDP, the other phases have points for which $\Omega_{c} h^{2}=\left(\Omega_{c} h^{2}\right)_{\exp }$ for all values above $125 / 2 \mathrm{GeV}$. The DDP has a DM mass region between about 100 and $500 \mathrm{GeV}$ where did not find any parameter points that saturate the relic density and extra 

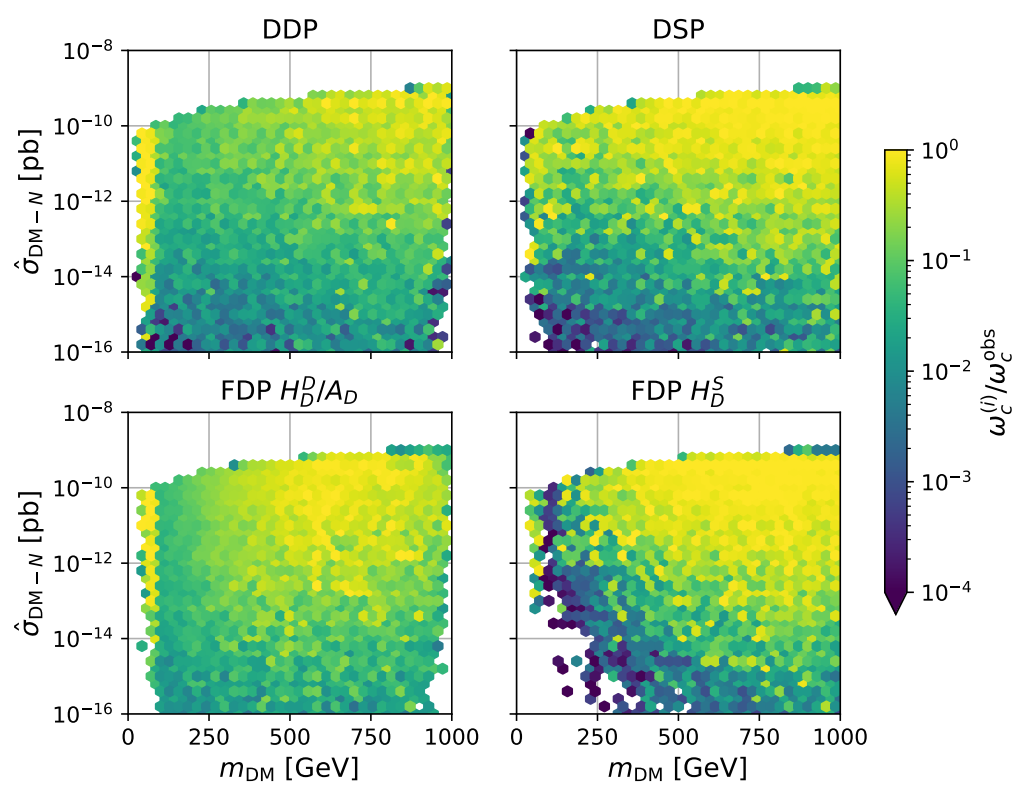

Figure 3. Nucleon-DM cross section, $\hat{\sigma}_{\mathrm{DM}-\mathrm{N}}$, as a function of the DM mass with all the constraints previously discussed. The colour code represents the fraction of the DM relic density, where the upper limit is the central value measured by Planck plus $2 \times 1 \sigma$. On the left upper plot we show the DDP and take the lightest dark sector particle to be the dark matter candidate; on the upper right we show the DSP with the corresponding dark matter candidate. The lower plots show the FDP: here the DM particle is either $H_{D}^{D}$ or $A_{D}$ on the left and $H_{D}^{S}$ on the right; note that since the two symmetries are conserved separately there are always two dark matter candidates in the FDP.

DM candidates are needed. This is in line with previous results (see refs. [72-75]) where it was reported that for the Inert doublet Model, the dark matter relic density cannot be saturated for DM masses between about 75 and $500 \mathrm{GeV}$.

\section{The different phases at the LHC and future colliders}

The different phases of the N2HDM lead to different phenomenology at the LHC and at future colliders. There are obvious differences that would immediately exclude some of them. The discovery of a charged Higgs boson would immediately exclude the DDP and the FDP. The discovery of three extra neutral scalars in the visible sector would exclude all phases except the broken phase. However, the best chances we have to probe the different phases are the $125 \mathrm{GeV}$ Higgs rates measurements and perhaps the search for an extra neutral scalar.

\section{$5.1 \quad h_{125}$ coupling measurements}

Let us start with the $125 \mathrm{GeV}$ Higgs coupling measurements. All phases have an alignment limit, that is, there is a set of values for which the $h_{125}$ couplings to fermions and gauge bosons are exactly the SM ones. Hence, in order to be able to distinguish between the phases we need a decay with a new contribution from a coupling which does not exist the 


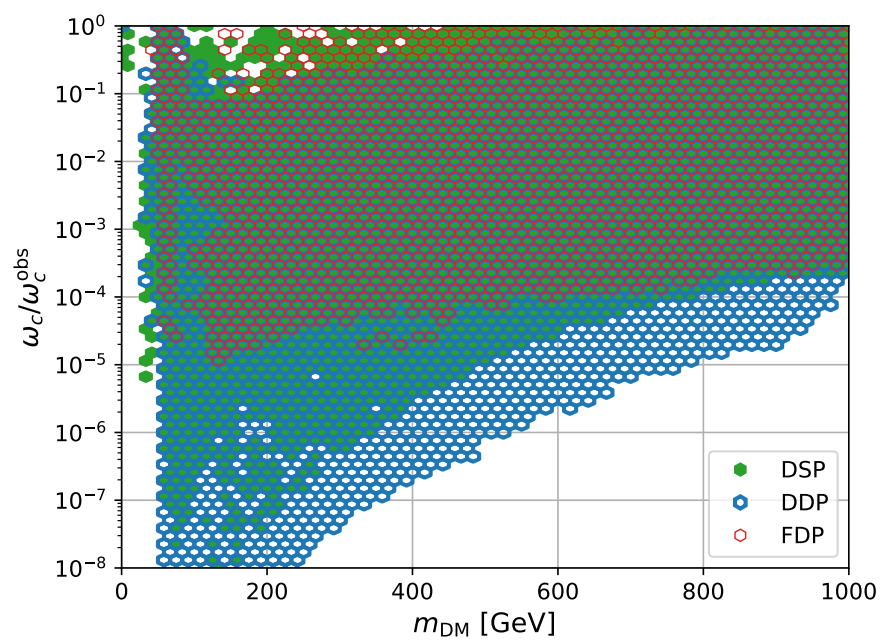

Figure 4. Fraction of the DM relic density as a function of the DM mass for the three DM phases. For the FDP, we show the mass of the dark matter candidate that gives the largest contribution to the relic density.

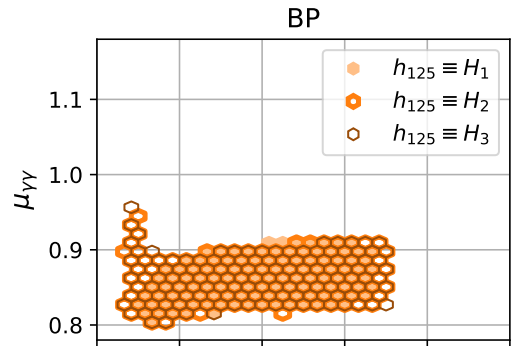

DSP

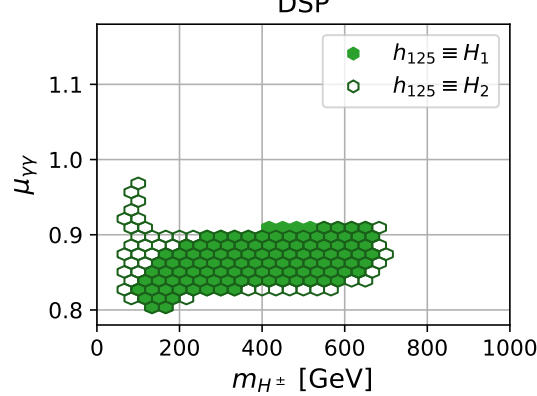

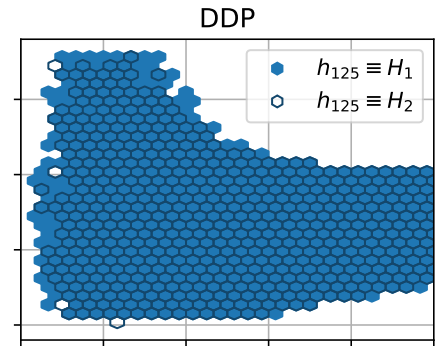

FDP

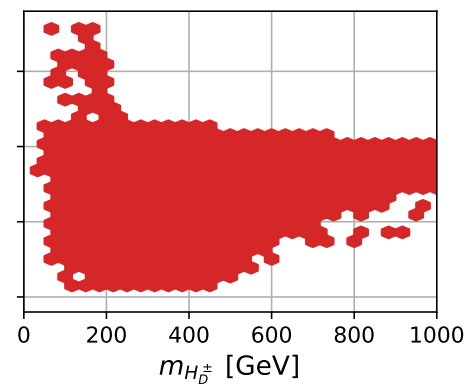

Figure 5. $\mu_{\gamma \gamma}$ as a function of the charged Higgs mass for the four N2HDM phases.

$\mathrm{SM}$ and originates from the Higgs potential. Such is the case of the $h_{125} \rightarrow \gamma \gamma$ decay, which has a contribution from the $h_{125} H^{+} H^{-}$vertex. In figure 5 we present $\mu_{\gamma \gamma}$ as a function of the charged Higgs mass for the four N2HDM phases. In the BP and DSP phases, which are the ones with charged scalars in the visible sector, the value of $\mu_{\gamma \gamma}$ is always below 0.98 and for charged Higgs masses above $150 \mathrm{GeV}$ the value is about 0.9 or below. The reason for the low values of $\mu_{\gamma \gamma}$ is due to setting $m_{12}^{2}=0$ (this is the soft breaking term that is usually included in the broken phase of the 2HDM and in that of the N2HDM). 
In this limit, the contribution from the $h_{125} H^{+} H^{-}$vertex, close to the alignment limit, is always negative, reducing the diphoton branching ratio of $h_{125}$ relative to its SM value. In the DDP and FDP the same vertex is proportional to the free parameter $m_{22}^{2}$, allowing for both negative and positive contributions. Therefore, the freedom in the coupling is lost due to $m_{12}^{2}=0$ in the visible phases, while in the dark phases the free mass parameter leads to a weaker constraint.

The presently measured value of $\kappa_{\gamma}=\sqrt{\Gamma\left(h_{\mathrm{NEW}} \rightarrow \gamma \gamma\right) / \Gamma\left(h_{\mathrm{SM}} \rightarrow \gamma \gamma\right)}$ is $0.97 \pm 0.07$ (at $1 \sigma)[76]$ while the HL-LHC $68 \%$ probability sensitivity to the same coupling modifier ranges from \pm 0.023 to \pm 0.016 [77]. This means that if by the end of the LHC high luminosity run the central value of the branching ratio of the Higgs boson to two photons is very close to the SM value and taking into account the predicted errors it is likely that the $\mathrm{BP}$ and the DSP will be excluded. The only possible exception is the light charged Higgs region which on the other hand will also be much more constrained by the end of the high luminosity phase by direct searches for charged Higgs bosons.

\subsection{Search for new scalars}

As previously discussed, there are some particularities that are specific to each model. The FDP can only be distinguished from the SM through the amount of missing energy in collider dark matter searches because it contains no new particles in the visible sector. Charged Higgs bosons in the visible sector are only possible in the BP and in the DSP. In order to distinguish these two phases one would need to look again into the amount of missing energy in searches for dark matter events at colliders. A feature that all of the phases except the FDP have in common is the existence of at least one additional, visible neutral scalar.

In figure 6 we show the production cross section for the non-SM like neutral Higgs with subsequent decay to $\tau^{+} \tau^{-}$(left) and $\gamma \gamma$ (right). In the phases where we have more than one visible scalar, we take all possibilities into account, that is, all CP-even scalars are considered. The decay to $\tau^{+} \tau^{-}$is chosen because it represents the general behaviour of the decays to fermions and the $b \bar{b}$ final state is much harder to resolve due to the background. The most relevant features of fermion final states are as follows. Below $m_{h_{125}} / 2$ the BP accommodates the largest possible rates because decays of the Higgs to dark matter are not possible in this phase. Still, in the DDP values of the cross section as large as $1 \mathrm{pb}$ are still possible. On the other hand the DDP has less freedom in the visible sector and therefore cross sections for masses above about $230 \mathrm{GeV}$ are already below $0.1 \mathrm{fb}$. Above $m_{\phi} / 2$ the $\mathrm{BP}$ and DSP are almost indistinguishable because their visible sectors are very similar to a $2 \mathrm{HDM}$, a feature that is reinforced by the tight constraints on the $h_{125}$ couplings and existing constraints from Higgs searches.

On the right plot of figure 6 we can see the decays to $\gamma \gamma$. In this case the DDP allows for substantially larger cross sections than the other phases that can even go up to $1 \mathrm{pb}$ for masses below $100 \mathrm{GeV}$. Note that although the DDP has less freedom in the visible sector it has more freedom in the dark sector and this is reflected in the couplings of the dark charged Higgs boson to the visible scalars. This can not only lead to the previously discussed large effects in $\mu_{\gamma \gamma}$ for $h_{125}$ but can also significantly enhance the $p p \rightarrow \phi \rightarrow \gamma \gamma$ 

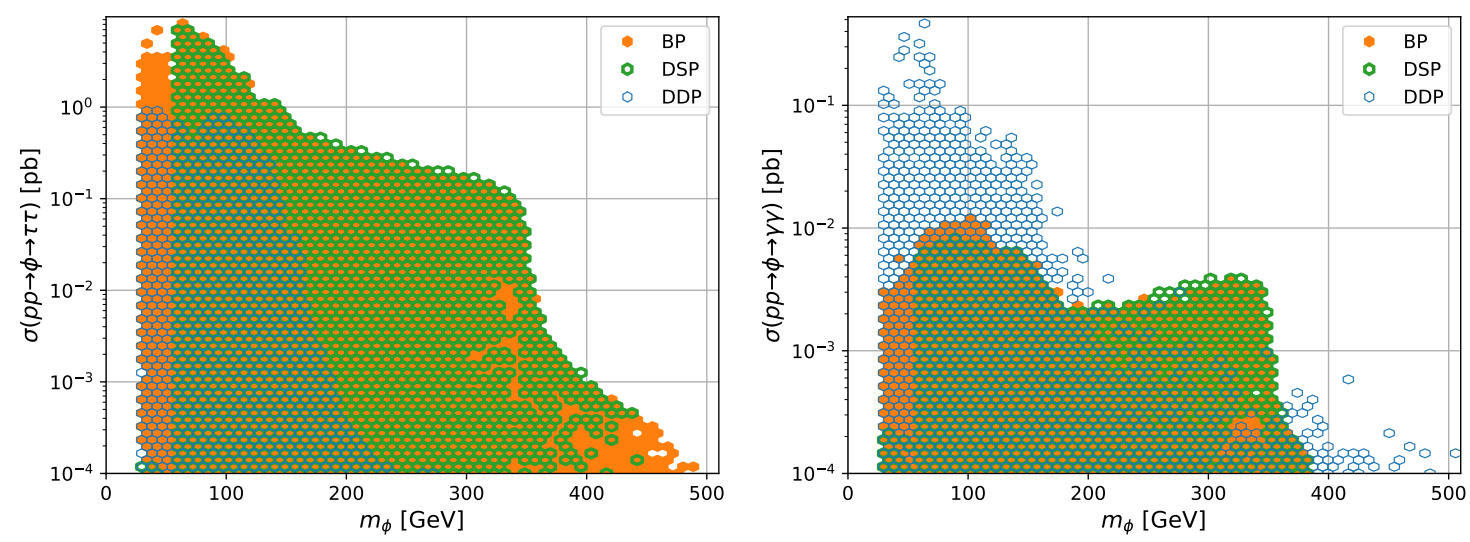

Figure 6. Production cross section for any of the non-SM like Higgs with subsequent decay to $\tau^{+} \tau^{-}$(left) and $\gamma \gamma$ (right) as a function of the scalar mass, for the BP, DSP and DDP. $\phi$ stands for any of the CP-even scalars in each phase, other than the $125 \mathrm{GeV}$ one.
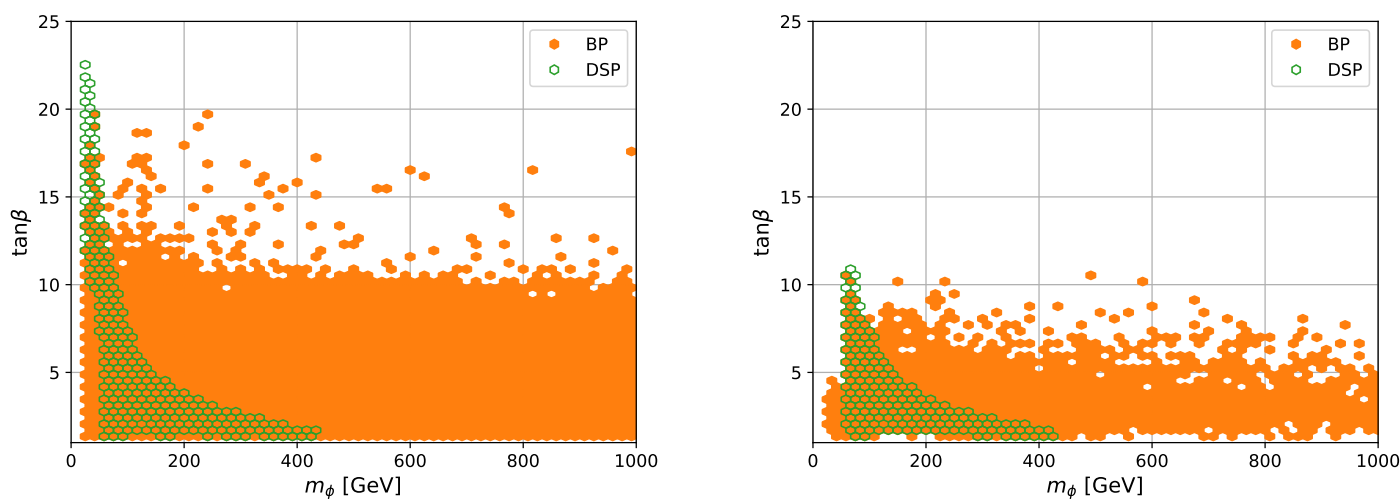

Figure 7. $\tan \beta$ as a function of the mass of any of the neutral non-SM like Higgs bosons with the present constraints (left) and taking the value of $c\left(h_{125} V V\right)$ to be 1 within $10^{-3}$ (right), for the BP and DSP. Again, $\phi$ stands for any of the CP-even scalars in each phase, other than the $125 \mathrm{GeV}$ one.

cross sections shown here. If such a signal is seen with rates above $10^{-2} \mathrm{pb}$ all phases except for the DDP would be excluded.

Let us finally comment on the behaviour of the model very close to the alignment limit. As shown in ref. [78], the $2 \mathrm{HDM}$ with an exact $\mathbb{Z}_{2}^{(1)}$ symmetry, and in the alignment limit where $\sin (\beta-\alpha)=1$ (or more generally $c\left(h_{125} V V\right)=1$ ), always has a value of $\tan \beta \lesssim 6$. In that reference they conclude that the limit arises from a combination of theoretical constraints together with taking the alignment limit. The left panel of figure 7 shows, for the BP and the DSP, $\tan \beta$ as a function of the mass of any of the CP-even, neutral scalars other than $h_{125}$, with all present experimental and theoretical constraints taken into account (note that there is no $\tan \beta$ in the DDP). The right panel is the same plot as the one on the left with the extra constraint of forcing $c\left(h_{125} V V\right)$ to be within 
$10^{-3}$ of the value 1 . Hence, although we have more freedom in our model because we have an extra singlet field, figure 7 shows that the allowed value of $\tan \beta$ is reduced as we approach the alignment limit. This has important consequences to corner the model using all experimental data. As an example, the experimental searches for charged Higgs bosons include the vertex $t b H^{ \pm}$which, in Yukawa sectors of Type I, is always proportional to $1 / \tan \beta$. Therefore, it will be very hard to access even the light charged Higgs for very large values of $\tan \beta$. However, with the restriction from the right plot of figure 7 , moving close to alignment reduces the allowed value of $\tan \beta$. Hence, if $\tan \beta$ is not too large it is more likely that the charged Higgs production cross section will be within experimental reach. The more constraints we can find from other sources the closer we will be to exclude a given phase.

\section{Conclusions}

In this work we have studied the four phases of the N2HDM - three of which have dark matter candidates. For the phases to be comparable, we have considered Yukawa sectors of type I and set $m_{12}^{2}=0$. The absence of this term makes the scalar potential correspond to an Inert Doublet Model extended by a real singlet field. The different phases have the same scalar potential and degrees of freedom but the fields in the dark sector vary from the FDP where all the extra degrees of freedom are in dark sector to the BP which has no dark matter candidate. The analysis of the vacuum structure of the four phases has shown an interesting behaviour of the possible neutral minima. We have shown that if a minimum in the BP or FDP exists, it is the global minimum of the theory. In that case all other stationary points of different phases lie above it and are saddle points. However, the same is not true for minima in the DDP and DSP - they can coexist in the potential, and neither is guaranteed to be deeper than the other.

Our main goal was to understand if the different phases could be probed and distinguished by combining the available experimental data and the one from future searches at colliders with that from dark matter experiments. We have generated samples of points for each phase which take into account the most up-to-date experimental data and also all relevant theoretical constraints. From the dark matter point of view, and in particular the direct detection bounds, all phases have valid points all the way to the neutrino floor. Hence, future direct detection experiments will not play a major role in constraining the parameter space of the model. As for dark matter relic density, all except the Dark Doublet Phase, have candidates for dark matter that saturate the relic density for a large range of dark masses. The DDP behaves very much like the Inert Doublet Model where, as previously discussed, the relic density cannot be saturated for dark matter masses between about $100 \mathrm{GeV}$ and $500 \mathrm{GeV}$. We have then looked for the effect of the Higgs coupling measurements and for the search for new particles at the LHC. Our main conclusions on what can we learn from the LHC are as follows:

- Finding a charged Higgs would single out the BP and DSP, while the discovery of any new neutral scalar would exclude the FDP. 
- Visible and dark sector charged Higgs bosons have very different impacts on the decays of the neutral scalars into $\gamma \gamma$. Visible $H^{ \pm}$always suppress $\mu_{\gamma \gamma}$ compared to the SM, while dark $H_{D}^{ \pm}$have more freedom in their couplings and could enhance or suppress the rate. As a result, a measurement of $\mu_{\gamma \gamma}$ at the end of the HL-LHC or future collider could very well exclude the BP and the DSP.

- In case a new scalar is found there are regions of parameter space where the 3 phases, BP, DDP and DSP could be distinguished in the decay to $\tau^{+} \tau^{-}$. Due to the dark charged Higgs, the DDP can predict very large rates for a new scalar decaying into $\gamma \gamma$ and may be probed there.

- If nothing is discovered and the $125 \mathrm{GeV}$ Higgs couplings are very close to the SM values, the FDP will always remain a possibility.

\section{A Dark doublet phase}

In this section, we present for the DDP the relation between the Lagrangian parameters and the physical parameters. First, the physical masses can be written as

$$
\begin{aligned}
m_{H_{1}}^{2} & =v^{2} \cos ^{2} \alpha \lambda_{1}+v_{s}^{2} \sin ^{2} \alpha \lambda_{6}+2 v v_{s} \sin \alpha \cos \alpha \lambda_{7}, \\
m_{H_{2}}^{2} & =v^{2} \sin ^{2} \alpha \lambda_{1}+v_{s}^{2} \cos ^{2} \alpha \lambda_{6}-2 v v_{s} \sin \alpha \cos \alpha \lambda_{7}, \\
m_{H_{D}}^{2} & =\frac{1}{2}\left(2 m_{22}^{2}+v^{2}\left(\lambda_{3}+\lambda_{4}+\lambda_{5}\right)+v_{s}^{2} \lambda_{8}\right), \\
m_{A_{D}}^{2} & =\frac{1}{2}\left(2 m_{22}^{2}+v^{2}\left(\lambda_{3}+\lambda_{4}-\lambda_{5}\right)+v_{s}^{2} \lambda_{8}\right), \\
m_{H_{D}^{ \pm}}^{2} & =\frac{1}{2}\left(2 m_{22}^{2}+v^{2} \lambda_{3}+v_{s}^{2} \lambda_{8}\right),
\end{aligned}
$$

which leads to the following relations between the parameters

$$
\begin{aligned}
\lambda_{1} & =\frac{1}{v^{2}}\left(\sum_{i} m_{H_{i}}^{2} \mathcal{R}_{i 1}^{2}\right), \\
\lambda_{3} & =\frac{1}{v^{2}}\left(2\left(m_{H_{D}^{ \pm}}^{2}-m_{22}^{2}\right)-v_{s}^{2} \lambda_{8}\right), \\
\lambda_{4} & =\frac{1}{v^{2}}\left(m_{A_{D}}^{2}+m_{H_{D}}^{2}-2 m_{H^{ \pm}}^{2}\right), \\
\lambda_{5} & =\frac{1}{v^{2}}\left(m_{H_{D}}^{2}-m_{A_{D}}^{2}\right), \\
\lambda_{6} & =\frac{1}{v_{s}^{2}}\left(\sum_{i} m_{H_{i}}^{2} \mathcal{R}_{i 3}^{2}\right), \\
\lambda_{7} & =\frac{1}{v v_{s}}\left(\sum_{i} m_{H_{i}}^{2} \mathcal{R}_{i 1} \mathcal{R}_{i 3}\right),
\end{aligned}
$$

where $\mathcal{R}_{i j}$ is the $i, j$ element of the mixing matrix in eq. (2.22). The parameters $m_{22}^{2}$, $\lambda_{2}$ and $\lambda_{8}$ cannot be expressed through physical parameters and thus remain independent parameters in the physical parameter set of the DDP. 


\section{A.1 Triple-Higgs couplings}

The triple-Higgs couplings $g\left(X_{i} X_{j} X_{k}\right)$ in the DDP are defined as,

$$
g\left(X_{i} X_{j} X_{k}\right)=\frac{\partial^{3} \mathcal{L}}{\partial X_{i} \partial X_{j} \partial X_{k}}
$$

with $X_{i / j / k} \in\left\{H_{1}, H_{2}, H_{D}, A_{D}, H_{D}^{ \pm}\right\}$. All couplings with an odd number of dark Higgs bosons vanish due to the conserved dark parity. The non-zero triple-Higgs couplings are the following, where the indices $i, j$ can only be $\{1,2\}$ and denote the visible CP-even Higgs bosons $H_{1}$ or $H_{2}$, respectively:

$$
\begin{aligned}
g\left(H_{i} H_{i} H_{i}\right)= & 3 \lambda_{1} v \mathcal{R}_{i 1}^{3}+3 \lambda_{6} v_{s} \mathcal{R}_{i 3}^{3} \\
& +3 \lambda_{7}\left(v \mathcal{R}_{i 1} \mathcal{R}_{i 3}^{2}+v_{s} \mathcal{R}_{i 3} \mathcal{R}_{i 1}^{2}\right) \\
g\left(H_{i} H_{j} H_{j}\right)= & 3 \lambda_{1} v \mathcal{R}_{i 1} \mathcal{R}_{j 1}^{2}+3 \lambda_{6} v_{s} \mathcal{R}_{i 3} \mathcal{R}_{j 3}^{2} \\
& +\lambda_{7}\left[v\left(\mathcal{R}_{i 1} \mathcal{R}_{j 3}^{2}+2 \mathcal{R}_{i 3} \mathcal{R}_{j 1} \mathcal{R}_{j 3}\right)\right. \\
& \left.+v_{s}\left(\mathcal{R}_{i 3} \mathcal{R}_{j 1}^{2}+2 \mathcal{R}_{i 1} \mathcal{R}_{j 1} \mathcal{R}_{j 3}\right)\right] \\
g\left(H_{i} H_{D} H_{D}\right)= & \frac{2}{v}\left(m_{H_{D}}^{2}-m_{22}^{2}\right) \mathcal{R}_{i 1}+\lambda_{8} \frac{v_{s}}{v}\left(v \mathcal{R}_{i 3}-v_{s} \mathcal{R}_{i 1}\right) \\
g\left(H_{i} H_{D}^{+} H_{D}^{-}\right)= & \frac{2}{v}\left(m_{H_{D}^{ \pm}}^{2}-m_{22}^{2}\right) \mathcal{R}_{i 1}+\lambda_{8} \frac{v_{s}}{v}\left(v \mathcal{R}_{i 3}-v_{s} \mathcal{R}_{i 1}\right), \\
g\left(H_{i} A_{D} A_{D}\right)= & \frac{2}{v}\left(m_{A_{D}}^{2}-m_{22}^{2}\right) \mathcal{R}_{i 1}+\lambda_{8} \frac{v_{s}}{v}\left(v \mathcal{R}_{i 3}-v_{s} \mathcal{R}_{i 1}\right)
\end{aligned}
$$

\section{B Dark singlet phase}

In this section, we present for the DSP the formulae for the masses and the relation between the gauge basis and the physical basis. The expressions for the masses are

$$
\begin{aligned}
m_{H_{1}}^{2}= & \frac{m_{12}^{2}}{v_{1} v_{2}}\left(v_{1} \cos \alpha+v_{2} \sin \alpha\right)^{2} \\
& +\lambda_{1} v_{1}^{2} \cos ^{2} \alpha+\lambda_{2} v_{2}^{2} \sin ^{2} \alpha-2 \lambda_{345} v_{1} v_{2} \cos \alpha \sin \alpha \\
m_{H_{2}}^{2}= & \frac{m_{12}^{2}}{v_{1} v_{2}}\left(v_{1} \sin \alpha-v_{2} \cos \alpha\right)^{2} \\
& +\lambda_{1} v_{1}^{2} \cos ^{2} \alpha+\lambda_{2} v_{2}^{2} \sin ^{2} \alpha+2 \lambda_{345} v_{1} v_{2} \cos \alpha \sin \alpha, \\
m_{H_{D}}^{2}= & \frac{1}{2}\left(2 m_{s}^{2}+v_{1}^{2} \lambda_{7}+v_{2}^{2} \lambda_{8}\right), \\
m_{A}^{2}= & -v^{2} \lambda_{5}+\frac{m_{12}^{2}}{s_{\beta} c_{\beta}}, \\
m_{H^{ \pm}}^{2}= & -\frac{1}{2} v^{2}\left(\lambda_{4}+\lambda_{5}\right)+\frac{m_{12}^{2}}{s_{\beta} c_{\beta}} .
\end{aligned}
$$


The relations between the two sets of parameters are

$$
\begin{aligned}
m_{S}^{2} & =-\frac{1}{2}\left(v_{1}^{2} \lambda_{7}+v_{2}^{2} \lambda_{8}-2 m_{H_{D}}\right), \\
\lambda_{1} & =\frac{1}{v^{2} c_{\beta}^{2}}\left[\left(\sum_{i} m_{H_{i}}^{2} \mathcal{R}_{i 1}^{2}\right)-m_{12}^{2} \frac{s_{\beta}}{c_{\beta}}\right], \\
\lambda_{2} & =\frac{1}{v^{2} s_{\beta}^{2}}\left[\left(\sum_{i} m_{H_{i}}^{2} \mathcal{R}_{i 2}^{2}\right)-m_{12}^{2} \frac{c_{\beta}}{s_{\beta}}\right], \\
\lambda_{3} & =\frac{1}{v^{2} c_{\beta} s_{\beta}}\left[\left(\sum_{i} m_{H_{i}}^{2} \mathcal{R}_{i 1} \mathcal{R}_{i 2}\right)-m_{12}^{2}\right]+\frac{2}{v^{2}} m_{H^{ \pm}}^{2}, \\
\lambda_{4} & =\frac{1}{v^{2}}\left(m_{A}^{2}-2 m_{H^{ \pm}}^{2}\right)+\frac{1}{v^{2} c_{\beta} s_{\beta}} m_{12}^{2}, \\
\lambda_{5} & =-\frac{1}{v^{2}} m_{A}^{2}+\frac{1}{v^{2} c_{\beta} s_{\beta}} m_{12}^{2},
\end{aligned}
$$

where $\mathcal{R}_{i j}$ is the $i, j$ element of the mixing matrix in eq. (2.32). The parameters $\lambda_{6}, \lambda_{7}$ and $\lambda_{8}$ cannot be expressed through physical parameters and thus remain independent parameters in the physical parameter set of the DSP.

\section{B.1 Triple-Higgs couplings}

We now present the triple-Higgs couplings $g\left(X_{i} X_{j} X_{k}\right)$ in the DSP. The definition of the coupling $g\left(X_{i} X_{j} X_{k}\right)$ is given in eq. (A.3) with $X_{i / j / k} \in\left\{H_{1}, H_{2}, H_{D}, A, H^{ \pm}\right\}$. All couplings with an odd number of $H_{D}$ vanish due to the conserved dark parity. The non-zero tripleHiggs couplings — with $i, j$ again reserved for the visible sector Higgs bosons - are

$$
\begin{aligned}
g\left(H_{i} H_{i} H_{i}\right)= & 3 v\left[c_{\beta}\left(\mathcal{R}_{i 1}^{3} \lambda_{1}+\mathcal{R}_{i 1} \mathcal{R}_{i 2}^{2} \lambda_{345}\right)+s_{\beta}\left(\mathcal{R}_{i 2}^{3} \lambda_{2}+\mathcal{R}_{i 2} \mathcal{R}_{i 1}^{2} \lambda_{345}\right)\right] \\
g\left(H_{i} H_{j} H_{j}\right)= & v\left[c_{\beta}\left(3 \mathcal{R}_{i 1} \mathcal{R}_{j 1}^{2} \lambda_{1}+\left(3 \mathcal{R}_{i 2} \mathcal{R}_{j 1} \mathcal{R}_{j 2}+\mathcal{R}_{i 1}\right) \lambda_{345}\right)\right. \\
& \left.+v s_{\beta}\left(3 \mathcal{R}_{i 2} \mathcal{R}_{j 2}^{2} \lambda_{2}+\left(3 \mathcal{R}_{i 1} \mathcal{R}_{j 1} \mathcal{R}_{j 2}+\mathcal{R}_{i 2}\right) \lambda_{345}\right)\right] \\
g\left(H_{i} A A\right)= & v\left[c_{\beta}\left(c_{\beta} s_{\beta} \mathcal{R}_{i 2}\left(\lambda_{2}-2 \lambda_{5}\right)+c_{\beta}^{2} \mathcal{R}_{i 1} \lambda_{34-5}\right)\right. \\
& \left.+s_{\beta}\left(c_{\beta} s_{\beta} \mathcal{R}_{i 2}\left(\lambda_{1}-2 \lambda_{5}\right)+s_{\beta}^{2} \mathcal{R}_{i 2} \lambda_{34-5}\right)\right] \\
g\left(H_{i} H^{+} H^{-}\right)= & v\left[c_{\beta}\left(s_{\beta}^{2} \mathcal{R}_{i 1} \lambda_{1}+c_{\beta}^{2} \mathcal{R}_{i 1} \lambda_{3}-c_{\beta} s_{\beta} \mathcal{R}_{i 2}\left(\lambda_{4}+\lambda_{5}\right)\right)\right. \\
& \left.+s_{\beta}\left(c_{\beta}^{2} \mathcal{R}_{i 2} \lambda_{2}+s_{\beta}^{2} \mathcal{R}_{i 2} \lambda_{3}-c_{\beta} s_{\beta} \mathcal{R}_{i 1}\left(\lambda_{4}+\lambda_{5}\right)\right)\right] \\
g\left(H_{i} H_{D} H_{D}\right)= & v\left[c_{\beta} \mathcal{R}_{i, 1} \lambda_{7}+s_{\beta} \mathcal{R}_{i, 2} \lambda_{8}\right]
\end{aligned}
$$

\section{Acknowledgments}

We acknowledge discussions with Igor Ivanov, Tania Robens and Dorota Sokolowska. $\mathrm{PF}$ and RS are supported by the Portuguese Foundation for Science and Technology (FCT), Contracts UIDB/00618/2020, UIDP/00618/2020, PTDC/FIS-PAR/31000/2017 
and CERN/FIS-PAR/0002/2017, and by the HARMONIA project, contract UMO2015/18/M/ST2/00518. JW has been funded by the European Research Council (ERC) under the European Union's Horizon 2020 research and innovation programme, grant agreement No 668679. MM is supported by the BMBF-Project 05H18VKCC1.

Open Access. This article is distributed under the terms of the Creative Commons Attribution License (CC-BY 4.0), which permits any use, distribution and reproduction in any medium, provided the original author(s) and source are credited.

\section{References}

[1] ATLAS collaboration, Observation of a new particle in the search for the Standard Model Higgs boson with the ATLAS detector at the LHC, Phys. Lett. B 716 (2012) 1 [arXiv: 1207.7214] [INSPIRE].

[2] CMS collaboration, Observation of a New Boson at a Mass of $125 \mathrm{GeV}$ with the CMS Experiment at the LHC, Phys. Lett. B 716 (2012) 30 [arXiv:1207.7235] [InSPIRE].

[3] G. Bertone, D. Hooper and J. Silk, Particle dark matter: Evidence, candidates and constraints, Phys. Rept. 405 (2005) 279 [hep-ph/0404175] [INSPIRE].

[4] Planck collaboration, Planck 2015 results. XIII. Cosmological parameters, Astron. Astrophys. 594 (2016) A13 [arXiv:1502.01589] [INSPIRE].

[5] Particle Data Group collaboration, Review of Particle Physics, Chin. Phys. C 40 (2016) 100001 [INSPIRE].

[6] Planck collaboration, Planck 2015 results. I. Overview of products and scientific results, Astron. Astrophys. 594 (2016) A1 [arXiv:1502.01582] [INSPIRE].

[7] V. Silveira and A. Zee, Scalar phantoms, Phys. Lett. B 161 (1985) 136 [InSPIRE].

[8] J. McDonald, Gauge singlet scalars as cold dark matter, Phys. Rev. D 50 (1994) 3637 [hep-ph/0702143] [INSPIRE].

[9] C.P. Burgess, M. Pospelov and T. ter Veldhuis, The Minimal model of nonbaryonic dark matter: A Singlet scalar, Nucl. Phys. B 619 (2001) 709 [hep-ph/0011335] [InSPIRE].

[10] N.G. Deshpande and E. Ma, Pattern of Symmetry Breaking with Two Higgs Doublets, Phys. Rev. D 18 (1978) 2574 [INSPIRE].

[11] E. Ma, Verifiable radiative seesaw mechanism of neutrino mass and dark matter, Phys. Rev. D 73 (2006) 077301 [hep-ph/0601225] [INSPIRE].

[12] R. Barbieri, L.J. Hall and V.S. Rychkov, Improved naturalness with a heavy Higgs: An Alternative road to LHC physics, Phys. Rev. D 74 (2006) 015007 [hep-ph/0603188] [INSPIRE].

[13] L. Lopez Honorez, E. Nezri, J.F. Oliver and M.H.G. Tytgat, The Inert Doublet Model: An Archetype for Dark Matter, JCAP 02 (2007) 028 [hep-ph/0612275] [INSPIRE].

[14] C.-Y. Chen, M. Freid and M. Sher, Next-to-minimal two Higgs doublet model, Phys. Rev. D 89 (2014) 075009 [arXiv: 1312.3949] [INSPIRE].

[15] A. Drozd, B. Grzadkowski, J.F. Gunion and Y. Jiang, Extending two-Higgs-doublet models by a singlet scalar field - the Case for Dark Matter, JHEP 11 (2014) 105 [arXiv:1408.2106] [INSPIRE]. 
[16] Y. Jiang, L. Li and R. Zheng, Boosted scalar confronting $750 \mathrm{GeV}$ di-photon excess, arXiv: 1605.01898 [INSPIRE].

[17] M. Muhlleitner, M.O.P. Sampaio, R. Santos and J. Wittbrodt, The N2HDM under Theoretical and Experimental Scrutiny, JHEP 03 (2017) 094 [arXiv: 1612.01309] [INSPIRE].

[18] M. Mühlleitner, M.O.P. Sampaio, R. Santos and J. Wittbrodt, Phenomenological Comparison of Models with Extended Higgs Sectors, JHEP 08 (2017) 132 [arXiv:1703.07750] [INSPIRE].

[19] J.F. Gunion, H.E. Haber, G.L. Kane and S. Dawson, The Higgs Hunter's Guide, vol. 80 (2000) [INSPIRE].

[20] P.M. Ferreira, M. Mühlleitner, R. Santos, G. Weiglein and J. Wittbrodt, Vacuum Instabilities in the N2HDM, JHEP 09 (2019) 006 [arXiv:1905.10234] [INSPIRE].

[21] P. Basler and M. Mühlleitner, BSMPT (Beyond the Standard Model Phase Transitions): A tool for the electroweak phase transition in extended Higgs sectors, Comput. Phys. Commun. 237 (2019) 62 [arXiv:1803.02846] [INSPIRE].

[22] P. Basler, M. Mühlleitner and J. Müller, Electroweak Phase Transition in Non-Minimal Higgs Sectors, JHEP 05 (2020) 016 [arXiv: 1912.10477] [INSPIRE].

[23] I. Engeln, Phenomenological comparison of the dark phases of the next-to-two-higgs-doublet model, MSc Thesis, Karlsruher Institut für Technologie, Karlsruhe Germany (2018).

[24] J. Velhinho, R. Santos and A. Barroso, Tree level vacuum stability in two Higgs doublet models, Phys. Lett. B 322 (1994) 213 [INSPIRE].

[25] P.M. Ferreira, R. Santos and A. Barroso, Stability of the tree-level vacuum in two Higgs doublet models against charge or CP spontaneous violation, Phys. Lett. B 603 (2004) 219 [Erratum ibid. 629 (2005) 114] [hep-ph/0406231] [INSPIRE].

[26] A. Barroso, P.M. Ferreira and R. Santos, Charge and CP symmetry breaking in two Higgs doublet models, Phys. Lett. B 632 (2006) 684 [hep-ph/0507224] [INSPIRE].

[27] C.C. Nishi, CP violation conditions in N-Higgs-doublet potentials, Phys. Rev. D 74 (2006) 036003 [Erratum ibid. 76 (2007) 119901] [hep-ph/0605153] [INSPIRE].

[28] M. Maniatis, A. von Manteuffel, O. Nachtmann and F. Nagel, Stability and symmetry breaking in the general two-Higgs-doublet model, Eur. Phys. J. C 48 (2006) 805 [hep-ph/0605184] [INSPIRE].

[29] I.P. Ivanov, Minkowski space structure of the Higgs potential in 2HDM, Phys. Rev. D 75 (2007) 035001 [Erratum ibid. 76 (2007) 039902] [hep-ph/0609018] [INSPIRE].

[30] A. Barroso, P.M. Ferreira and R. Santos, Neutral minima in two-Higgs doublet models, Phys. Lett. B 652 (2007) 181 [hep-ph/0702098] [INSPIRE].

[31] C.C. Nishi, The Structure of potentials with N Higgs doublets, Phys. Rev. D 76 (2007) 055013 [arXiv: 0706 . 2685] [INSPIRE].

[32] M. Maniatis, A. von Manteuffel and O. Nachtmann, CP violation in the general two-Higgs-doublet model: A Geometric view, Eur. Phys. J. C 57 (2008) 719 [arXiv:0707.3344] [INSPIRE].

[33] I.P. Ivanov, Minkowski space structure of the Higgs potential in 2HDM. II. Minima, symmetries, and topology, Phys. Rev. D 77 (2008) 015017 [arXiv:0710.3490] [INSPIRE]. 
[34] M. Maniatis, A. von Manteuffel and O. Nachtmann, A New type of CP symmetry, family replication and fermion mass hierarchies, Eur. Phys. J. C 57 (2008) 739 [arXiv:0711.3760] [INSPIRE].

[35] C.C. Nishi, Physical parameters and basis transformations in the Two-Higgs-Doublet model, Phys. Rev. D 77 (2008) 055009 [arXiv:0712.4260] [INSPIRE].

[36] M. Maniatis and O. Nachtmann, On the phenomenology of a two-Higgs-doublet model with maximal CP symmetry at the LHC, JHEP 05 (2009) 028 [arXiv:0901.4341] [INSPIRE].

[37] P.M. Ferreira, M. Maniatis, O. Nachtmann and J.P. Silva, CP properties of symmetry-constrained two-Higgs-doublet models, JHEP 08 (2010) 125 [arXiv:1004.3207] [INSPIRE].

[38] I.P. Ivanov and C.C. Nishi, Properties of the general NHDM. I. The Orbit space, Phys. Rev. D 82 (2010) 015014 [arXiv: 1004.1799] [INSPIRE].

[39] I.P. Ivanov and C.C. Nishi, Symmetry breaking patterns in 3HDM, JHEP 01 (2015) 021 [arXiv:1410.6139] [INSPIRE].

[40] I.P. Ivanov, M. Köpke and M. Mühlleitner, Algorithmic Boundedness-From-Below Conditions for Generic Scalar Potentials, Eur. Phys. J. C 78 (2018) 413 [arXiv:1802.07976] [inSPIRE].

[41] P.M. Ferreira, The vacuum structure of the Higgs complex singlet-doublet model, Phys. Rev. D 94 (2016) 096011 [arXiv:1607.06101] [INSPIRE].

[42] P.M. Ferreira and B.L. Gonçalves, Stability of neutral minima against charge breaking in the Higgs triplet model, JHEP 02 (2020) 182 [arXiv:1911.09746] [INSPIRE].

[43] R. Coimbra, M.O.P. Sampaio and R. Santos, ScannerS: Constraining the phase diagram of a complex scalar singlet at the LHC, Eur. Phys. J. C 73 (2013) 2428 [arXiv:1301.2599] [INSPIRE].

[44] M. Mühlleitner, M.O.P. Sampaio, R. Santos and J. Wittbrodt, ScannerS: Parameter Scans in Extended Scalar Sectors, arXiv:2007.02985 [INSPIRE].

[45] I. Engeln, M. Mühlleitner and J. Wittbrodt, N2HDECAY: Higgs Boson Decays in the Different Phases of the N2HDM, Comput. Phys. Commun. 234 (2019) 256 [arXiv: 1805.00966] [INSPIRE].

[46] M. Krause and M. Mühlleitner, ewN2HDECAY - A program for the Calculation of Electroweak One-Loop Corrections to Higgs Decays in the Next-to-Minimal Two-Higgs-Doublet Model Including State-of-the-Art QCD Corrections, arXiv:1904.02103 [INSPIRE].

[47] R.V. Harlander, S. Liebler and H. Mantler, SusHi: A program for the calculation of Higgs production in gluon fusion and bottom-quark annihilation in the Standard Model and the MSSM, Comput. Phys. Commun. 184 (2013) 1605 [arXiv:1212.3249] [INSPIRE].

[48] R.V. Harlander, S. Liebler and H. Mantler, SusHi Bento: Beyond NNLO and the heavy-top limit, Comput. Phys. Commun. 212 (2017) 239 [arXiv:1605.03190] [INSPIRE].

[49] R. Harlander, M. Mühlleitner, J. Rathsman, M. Spira and O. Stål, Interim recommendations for the evaluation of Higgs production cross sections and branching ratios at the LHC in the Two-Higgs-Doublet Model, arXiv:1312.5571 [INSPIRE]. 
[50] W.G. Hollik, G. Weiglein and J. Wittbrodt, Impact of Vacuum Stability Constraints on the Phenomenology of Supersymmetric Models, JHEP 03 (2019) 109 [arXiv: 1812.04644] [INSPIRE].

[51] ATLAS and CMS collaborations, Combined Measurement of the Higgs Boson Mass in pp Collisions at $\sqrt{s}=7$ and $8 \mathrm{TeV}$ with the ATLAS and CMS Experiments, Phys. Rev. Lett. 114 (2015) 191803 [arXiv:1503.07589] [INSPIRE].

[52] M.E. Peskin and T. Takeuchi, Estimation of oblique electroweak corrections, Phys. Rev. D 46 (1992) 381 [INSPIRE].

[53] W. Grimus, L. Lavoura, O.M. Ogreid and P. Osland, A Precision constraint on multi-Higgs-doublet models, J. Phys. G 35 (2008) 075001 [arXiv:0711.4022] [INSPIRE].

[54] W. Grimus, L. Lavoura, O.M. Ogreid and P. Osland, The Oblique parameters in multi-Higgs-doublet models, Nucl. Phys. B 801 (2008) 81 [arXiv:0802.4353] [INSPIRE].

[55] J. Haller, A. Hoecker, R. Kogler, K. Mönig, T. Peiffer and J. Stelzer, Update of the global electroweak fit and constraints on two-Higgs-doublet models, Eur. Phys. J. C 78 (2018) 675 [arXiv: 1803.01853] [INSPIRE].

[56] P. Bechtle, O. Brein, S. Heinemeyer, G. Weiglein and K.E. Williams, HiggsBounds: Confronting Arbitrary Higgs Sectors with Exclusion Bounds from LEP and the Tevatron, Comput. Phys. Commun. 181 (2010) 138 [arXiv:0811.4169] [InSPIRE].

[57] P. Bechtle, O. Brein, S. Heinemeyer, G. Weiglein and K.E. Williams, HiggsBounds 2.0.0: Confronting Neutral and Charged Higgs Sector Predictions with Exclusion Bounds from LEP and the Tevatron, Comput. Phys. Commun. 182 (2011) 2605 [arXiv:1102.1898] [inSPIRE].

[58] P. Bechtle et al., HiggsBounds - 4: Improved Tests of Extended Higgs Sectors against Exclusion Bounds from LEP, the Tevatron and the LHC, Eur. Phys. J. C 74 (2014) 2693 [arXiv: 1311.0055] [INSPIRE].

[59] P. Bechtle, S. Heinemeyer, O. Stål, T. Stefaniak and G. Weiglein, HiggsSignals: Confronting arbitrary Higgs sectors with measurements at the Tevatron and the LHC, Eur. Phys. J. C 74 (2014) 2711 [arXiv:1305.1933] [InSPIRE].

[60] G. Bélanger, F. Boudjema, A. Pukhov and A. Semenov, MicrOMEGAs 2.0: A Program to calculate the relic density of dark matter in a generic model, Comput. Phys. Commun. $\mathbf{1 7 6}$ (2007) 367 [hep-ph/0607059] [INSPIRE].

[61] G. Bélanger, F. Boudjema, A. Pukhov and A. Semenov, Dark matter direct detection rate in a generic model with MicrOMEGAs 2.2, Comput. Phys. Commun. 180 (2009) 747 [arXiv: 0803.2360] [INSPIRE].

[62] G. Bélanger et al., Indirect search for dark matter with MicrOMEGAs2.4, Comput. Phys. Commun. 182 (2011) 842 [arXiv: 1004.1092] [INSPIRE].

[63] G. Bélanger, F. Boudjema, A. Pukhov and A. Semenov, MicrOMEGAs_3: A program for calculating dark matter observables, Comput. Phys. Commun. 185 (2014) 960 [arXiv: 1305.0237] [INSPIRE].

[64] G. Bélanger, F. Boudjema, A. Pukhov and A. Semenov, MicrOMEGAs4.1: two dark matter candidates, Comput. Phys. Commun. 192 (2015) 322 [arXiv:1407.6129] [InSPIRE].

[65] D. Barducci et al., Collider limits on new physics within MicrOMEGAs_4.3, Comput. Phys. Commun. 222 (2018) 327 [arXiv:1606.03834] [INSPIRE]. 
[66] G. Bélanger, F. Boudjema, A. Goudelis, A. Pukhov and B. Zaldivar, MicrOMEGAs5.0 : Freeze-in, Comput. Phys. Commun. 231 (2018) 173 [arXiv:1801.03509] [INSPIRE].

[67] Planck collaboration, Planck 2018 results. VI. Cosmological parameters, arXiv:1807.06209 [INSPIRE].

[68] XENON collaboration, Dark Matter Search Results from a One Ton-Year Exposure of XENON1T, Phys. Rev. Lett. 121 (2018) 111302 [arXiv:1805.12562] [INSPIRE].

[69] ATLAS collaboration, Combination of searches for invisible Higgs boson decays with the ATLAS experiment, Phys. Rev. Lett. 122 (2019) 231801 [arXiv: 1904.05105] [INSPIRE].

[70] XENON collaboration, First Dark Matter Search Results from the XENON1T Experiment, Phys. Rev. Lett. 119 (2017) 181301 [arXiv:1705. 06655] [INSPIRE].

[71] J. Billard, L. Strigari and E. Figueroa-Feliciano, Implication of neutrino backgrounds on the reach of next generation dark matter direct detection experiments, Phys. Rev. D 89 (2014) 023524 [arXiv: 1307.5458] [INSPIRE].

[72] A. Arhrib, Y.-L.S. Tsai, Q. Yuan and T.-C. Yuan, An Updated Analysis of Inert Higgs Doublet Model in light of the Recent Results from LUX, PLANCK, AMS-02 and LHC, JCAP 06 (2014) 030 [arXiv:1310.0358] [INSPIRE].

[73] A. Ilnicka, M. Krawczyk and T. Robens, Inert Doublet Model in light of LHC Run I and astrophysical data, Phys. Rev. D 93 (2016) 055026 [arXiv:1508.01671] [INSPIRE].

[74] A. Belyaev, G. Cacciapaglia, I.P. Ivanov, F. Rojas-Abatte and M. Thomas, Anatomy of the Inert Two Higgs Doublet Model in the light of the LHC and non-LHC Dark Matter Searches, Phys. Rev. D 97 (2018) 035011 [arXiv:1612.00511] [INSPIRE].

[75] J. Kalinowski, W. Kotlarski, T. Robens, D. Sokolowska and A.F. Zarnecki, Benchmarking the Inert Doublet Model for $e^{+} e^{-}$colliders, JHEP 12 (2018) 081 [arXiv: 1809.07712] [INSPIRE].

[76] J. de Blas, O. Eberhardt and C. Krause, Current and Future Constraints on Higgs Couplings in the Nonlinear Effective Theory, JHEP 07 (2018) 048 [arXiv: 1803. 00939] [INSPIRE].

[77] M. Cepeda et al., Report from Working Group 2: Higgs Physics at the HL-LHC and HE-LHC, CERN Yellow Rep. Monogr. 7 (2019) 221 [arXiv:1902.00134] [INSPIRE].

[78] B. Gorczyca and M. Krawczyk, Tree-Level Unitarity Constraints for the SM-like 2HDM, arXiv:1112.5086 [INSPIRE]. 